\title{
AN ABELIAN EMBEDDING FOR MOORE SPECTRA
}

\author{
N. P. STRICKLAND
}

\begin{abstract}
We embed the category of Moore spectra as a full subcategory of an abelian category, and make some remarks about abelian embeddings of various other categories of spectra.
\end{abstract}

\section{INTRODUCTION}

Given any small additive category $\mathcal{I}$, we write $[\mathcal{I}, \mathrm{Ab}]$ for the category of additive functors $\mathcal{I} \rightarrow \mathrm{Ab}$. We will call such categories diagram categories.

Let Spectra denote the homotopy category of spectra (and maps of degree zero only), and let $\mathcal{C}$ be a full subcategory of Spectra (which need not be triangulated). By an embedding of $\mathcal{C}$ we mean a full and faithful additive functor from $\mathcal{C}$ to some diagram category.

In principle, such embeddings should be common. A theorem of Freyd [3] guarantees that every triangulated category can be embedded in an abelian category, and there are other theorems (the most famous due to Gabriel and Popescu [4) saying that abelian categories can be embedded in diagram categories under fairly mild conditions. However, explicit examples are rare. The best known cases are as follows:

(a) Let $\mathcal{C}_{0}$ be the category of rational spectra (those for which each homotopy group $\pi_{n}(X)$ is uniquely divisible). Let $\mathcal{I}_{0}$ be the category with object set $\mathbb{Z}$ and

$$
\mathcal{I}_{0}(n, m)= \begin{cases}\mathbb{Q} & \text { if } n=m \\ 0 & \text { otherwise }\end{cases}
$$

so $\left[\mathcal{I}_{0}, \mathrm{Ab}\right]$ is the category of graded rational vector spaces. Then we have an equivalence $\pi_{*}: \mathcal{C}_{0} \rightarrow$ $\left[\mathcal{I}_{0}, \mathrm{Ab}\right]$.

(b) Let $\mathcal{C}_{1}$ be the category of Eilenberg-MacLane spectra (those with $\pi_{k}(X)=0$ for $k \neq 0$ ). Then $\pi_{0}: \mathcal{C}_{1} \rightarrow \mathrm{Ab}$ is an equivalence.

(c) By a free Moore spectrum we mean a $(-1)$-connected spectrum $X$ such that $\pi_{0}(X)$ is free abelian and $H_{k}(X)=0$ for $k>0$. Let $\mathcal{C}_{2}$ be the category of free Moore spectra. One can check that any such spectrum is a wedge of copies of the sphere spectrum $S$, and that $\pi_{0}: \mathcal{C}_{2} \rightarrow$ FreeAb is an equivalence.

(d) Let $\mathcal{C}_{3}$ be the category of finite spectra. Let $\mathcal{I}_{3}$ be the category with object set $\mathbb{Z}$ and $\mathcal{I}_{3}(n, m)=$ $\pi_{m-n}(S)$, so $\left[\mathcal{I}_{3}, \mathrm{Ab}\right]$ is the category of graded modules over $\pi_{*}(S)$. We have a functor $\pi_{*}: \mathcal{C}_{3} \rightarrow$ $\left[\mathcal{I}_{3}, \mathrm{Ab}\right]$, and an old conjecture of Freyd suggests that this should be an embedding. However, there is no proof in sight.

(e) Say that a spectrum $X$ is $M U$-injective if it is a retract of $M U \wedge T$ for some $T$, and let $\mathcal{C}_{4}$ be the category of $M U$-injective spectra. Let $\mathcal{I}_{4}$ be the category with object set $\mathbb{Z}$ and $\mathcal{I}_{4}(n, m)=$ $\left[\Sigma^{-n} M U, \Sigma^{-m} M U\right]$. Define $F: \mathcal{C}_{4} \rightarrow\left[\mathcal{I}_{4}, \mathrm{Ab}\right]$ by $F(X)(n)=\pi_{0}\left(X \wedge \Sigma^{-n} M U\right)=M U_{n}(X)$. It is known that this is an embedding.

On the other hand, there are various known examples of subcategories $\mathcal{C}$ with functors $F: \mathcal{C} \rightarrow \mathcal{A}$ (for some diagram category $\mathcal{A})$ where the maps $F:[X, Y] \rightarrow \mathcal{A}(F X, F Y)$ are surjective and the kernel has a straightforward description in terms of well-understood invariants of $X$ and $Y$. It is thus natural to ask what is needed to improve such a functor to an embedding in some more complex diagram category.

In this note we will carry out this programme in the simplest possible case, that of Moore spectra. (We also have some comments about slightly larger categories.) The essentially equivalent case of Moore spaces of fixed dimension $d>1$ has also been discussed by Baues in [1, Section V.3a] (which we read before writing this paper) and [2, Chapter 1] (which was brought to our attention by a referee). It seems likely that our main theorem could be obtained by combining his results and spelling out various definitions more explicitly. However, we will instead give a direct approach with more details and context, and greater focus on making 
all constructions manifestly natural. The amount of work involved was a surprise to the author, at least. We now describe the main result.

Definition 1.1. A Moore spectrum is a spectrum $X$ such that $\pi_{k}(X)=0$ for $k<0$ and $H_{k}(X)=0$ for $k>0$. We write Moore for the category of Moore spectra (a full subcategory of the homotopy category of spectra).

Remark 1.2. It is traditional and convenient to use notation $S A$ or $M A$ for a Moore spectrum $X$ with $\pi_{0}(X) \simeq A$, but this suggests that $S A$ is a functor of $A$, which is not quite correct. As we are focussing here on questions of naturality we therefore prefer to avoid such notation.

Definition 1.3. A Moore diagram is a diagram $M$ of Abelian groups of the form

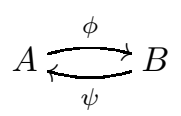

such that $\psi \phi=0$ and $\phi \psi=2.1_{B}$. We write MD for the category of Moore diagrams. We define forgetful functors $\alpha, \beta: \mathrm{MD} \rightarrow \mathrm{Ab}$ by $\alpha(M)=A$ and $\beta(M)=B$.

Note that if $M=(A, B, \phi, \psi)$ is a Moore diagram then $2 \phi=(\phi \psi) \phi=\phi(\psi \phi)=0$ and similarly $2 \psi=0$. This means that we have induced maps

$$
A / 2 \stackrel{\phi}{\rightarrow} B \stackrel{\psi}{\rightarrow} A[2]
$$

(where $A[2]$ means $\{a \in A \mid 2 a=0\}$ ). If this sequence is short exact, we say that $M$ is an exact Moore diagram. We write EMD for the full subcategory of exact Moore diagrams.

We will prove that Moore is equivalent to EMD. To define the relevant functor, let $S / 2$ denote the cofibre of the degree two self-map of the sphere spectrum $S$, so we have a cofibration sequence

$$
S \stackrel{2}{\rightarrow} S \stackrel{\rho}{\rightarrow} S / 2 \stackrel{\beta}{\rightarrow} S^{1} \stackrel{2}{\rightarrow} S^{1}
$$

Let $\eta$ be the Hopf map, which is the unique nontrivial element of $\pi_{1}(S)$.

Theorem 1.4. There is an equivalence $F:$ Moore $\rightarrow$ EMD sending $X \in$ Moore to the diagram

$$
[S, X] \underset{\rho^{*}}{\stackrel{(\eta \beta)^{*}}{\rightleftarrows}}[S / 2, X]
$$

Remark 1.5. Note here that $[S, X]$ is just another notation for $\pi_{0}(X)$. It can also be shown that there is a map $\zeta: S^{2} \rightarrow S / 2$ (of order 4 ) such that $\beta \zeta=\Sigma \eta$, and that the map $\zeta^{*}:[S / 2, X] \rightarrow \pi_{2}(X)$ is an isomorphism for Moore spectra $X$. (This will be proved as Lemma 5.16, ) Thus, the diagram above is isomorphic to the diagram

$$
\pi_{0}(X) \underset{\rho^{*}\left(\zeta^{*}\right)^{-1}}{\stackrel{\left(\eta^{2}\right)^{*}}{\rightleftarrows}} \pi_{2}(X)
$$

Theorem 1.4 will be proved in Section 5 .

\section{An Ext isomorphism}

It will be convenient to have the following result in hand before we start our main discussion.

Proposition 2.1. Let $U$ and $V$ be abelian groups. Then for any extension $E=(V \stackrel{i}{\rightarrow} M \stackrel{p}{\rightarrow} U)$ there is a well-defined homomorphism $\Phi(E): U[2] \rightarrow V / 2$ given by $\Phi(E)(u)=i^{-1}\left(2 p^{-1}(u)\right)+2 V$. This depends only on the equivalence class of the extension $E$, and the resulting map $\Phi: \operatorname{Ext}(U, V) / 2 \rightarrow \operatorname{Hom}(U[2], V / 2)$ is an isomorphism. Moreover, there is always an exact sequence

$$
V[2] \stackrel{i}{\longrightarrow} M[2] \stackrel{p}{\longrightarrow} U[2] \stackrel{\Phi(E)}{\longrightarrow} V / 2 \stackrel{i}{\longrightarrow} M / 2 \stackrel{p}{\longrightarrow} U / 2 .
$$


Proof. First, the homomorphism $\Phi(E)$ arises by applying the Snake Lemma to the diagram

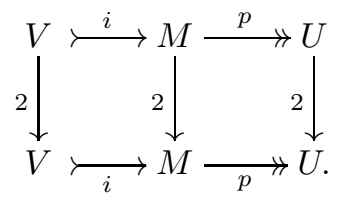

This makes it clear that $\Phi$ is well-defined, that it gives a six-term exact sequence as claimed, and that it is natural in the following sense: for homomorphisms $f: V \rightarrow V^{\prime}$ and $h: U^{\prime} \rightarrow U$, the map $\Phi\left(h^{*} f_{*} E\right)$ is the composite

$$
U^{\prime}[2] \stackrel{h}{\rightarrow} U[2] \stackrel{\Phi(E)}{\longrightarrow} V / 2 \stackrel{f}{\rightarrow} V^{\prime} / 2 .
$$

It is also clear that $\Phi\left(E \oplus E^{\prime}\right)=\Phi(E) \oplus \Phi\left(E^{\prime}\right)$. Now suppose we have two parallel extensions $E=(V \rightarrow$ $M \rightarrow U)$ and $E^{\prime}=\left(V \rightarrow M^{\prime} \rightarrow U\right)$. In this context we have a diagonal map $\Delta: U \rightarrow U \oplus U$ and a codiagonal map $\nabla: V \oplus V \rightarrow V$, so we can form the extension $E^{\prime \prime}=\nabla_{*} \Delta^{*}\left(E \oplus E^{\prime}\right)$. This operation is called the Baer sum, and it corresponds to addition in the group $\operatorname{Ext}(U, V)$. In this case the rule for $\Phi\left(h^{*} f_{*} E\right)$ just tells us that $\Phi\left(E^{\prime \prime}\right)=\Phi(E)+\Phi\left(E^{\prime}\right)$, so $\Phi$ gives a homomorphism $\operatorname{Ext}(U, V) \rightarrow \operatorname{Hom}(U[2], V / 2)$.

Now consider the case where $2 U=0$ and $2 V=0$, so $U[2]=U$ and $V / 2=V$ so we have a map $\Phi: \operatorname{Ext}(U, V) \rightarrow \operatorname{Hom}(U, V)$. Suppose that $\Phi(E)=0$. The Snake Lemma gives an exact sequence

$$
V \rightarrow M[2] \stackrel{p}{\rightarrow} U \stackrel{\Phi(E)}{\rightarrow} V \stackrel{i}{\rightarrow} M / 2 \rightarrow U
$$

but $\Phi(E)=0$ so the map $p: M[2] \rightarrow U$ is surjective. This can be regarded as an epimorphism of vector spaces over the field $\mathbb{Z} / 2$, so it has a section, and any such section gives a splitting of $E$, so $[E]=0$ in $\operatorname{Ext}(U, V)$. Thus, the map $\Phi: \operatorname{Ext}(U, V) \rightarrow \operatorname{Hom}(U, V)$ is injective.

Now choose a basis $\left\{u_{\alpha}\right\}_{\alpha \in I}$ for $U$ over $\mathbb{Z} / 2$. Let $\widetilde{U}$ be a free module over $\mathbb{Z} / 4$ with basis $\left\{\tilde{u}_{\alpha}\right\}_{\alpha \in I}$. We can then define an extension $E_{1}=\left(U \stackrel{i_{1}}{\longrightarrow} \widetilde{U} \stackrel{p_{1}}{\longrightarrow} U\right)$, where $i_{1}\left(u_{\alpha}\right)=2 \tilde{u}_{\alpha}$ and $p_{1}\left(\tilde{u}_{\alpha}\right)=u_{\alpha}$. We then find that $\Phi\left(E_{1}\right)$ is the identity map $1_{U}$. For any map $f: U \rightarrow V$ we deduce that $\Phi\left(f_{*} E_{1}\right)=f_{*} \Phi\left(E_{1}\right)=f$. This proves that $\Phi$ is surjective (and thus bijective) in the special case where $U$ and $V$ both have exponent two.

We now return to the general case. The standard six-term exact sequences for Ext tell us that functors of the form $\operatorname{Ext}(U,-)$ preserve right-exact sequences, and contravariant functors of the form $\operatorname{Ext}(-, V)$ convert left-exact sequences to right exact sequences. We can apply this to the sequences $U[2] \rightarrow U \stackrel{2}{\rightarrow} U$ and $V \stackrel{2}{\rightarrow} V \rightarrow V / 2$ to get a diagram with right-exact rows and columns as follows:

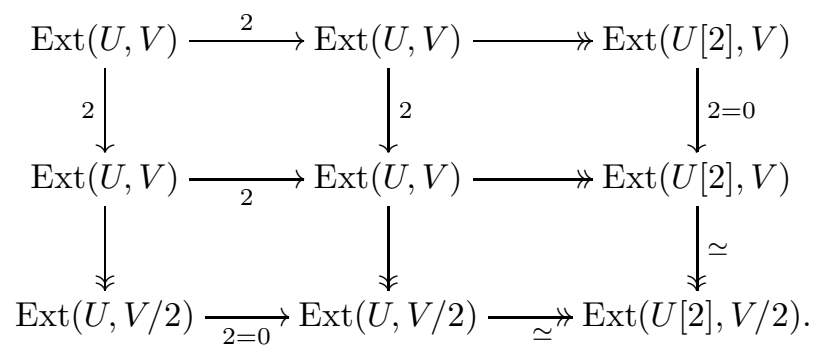

Right exactness means that all entries in the last row or column are isomorphic to $\operatorname{Ext}(U, V) / 2$, as claimed.

Remark 2.2. Note that if $2 U=0$ or $2 V=0$ then (as Ext is a biadditive functor) we have 2 . $\operatorname{Ext}(U, V)=0$. It follows that in these cases we just have $\operatorname{Ext}(U, V)=\operatorname{Hom}(U[2], V / 2)$.

Proposition 2.3. Suppose we have the solid part of the following diagram

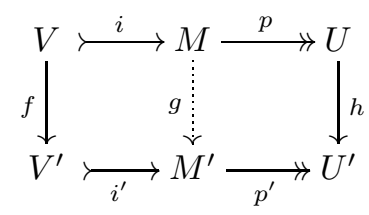


Suppose that the rows are exact, so they define extensions $E$ and $E^{\prime}$. Consider the following conditions:

(a) There is a map g making the whole diagram commute.

(b) $\left[f_{*} E\right]=\left[h^{*} E^{\prime}\right]$ in $\operatorname{Ext}\left(U, V^{\prime}\right)$

(c) The diagram

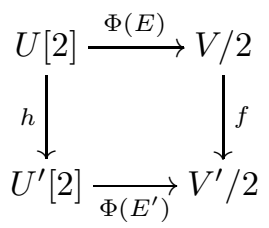

commutes.

Then (a) and (b) are equivalent, and they imply (c). The converse also holds provided that $\operatorname{Ext}\left(U, V^{\prime}\right)$ has exponent 2.

Proof. The equivalence of (a) and (b) is standard and straightforward. Condition (c) can be written as $f_{*} \Phi(E)=h^{*} \Phi\left(E^{\prime}\right)$ (in $\operatorname{Hom}\left(U[2], V^{\prime} / 2\right)$ ), so the naturality of $\Phi$ tells us that (b) implies (c). If $\operatorname{Ext}\left(U, V^{\prime}\right)$ has exponent two then the map $\Phi: \operatorname{Ext}\left(U, V^{\prime}\right) \rightarrow \operatorname{Hom}\left(U[2], V^{\prime} / 2\right)$ is an isomorphism so (c) also implies (b).

\section{Some Diagram CATEgories}

\section{Definition 3.1.}

(a) An $\eta$-diagram is just a diagram $A \stackrel{\eta}{\rightarrow} C$ of abelian groups such that $2 \eta=0$. We write ED for the category of $\eta$-diagrams. There are evident functors $\alpha, \gamma: \mathrm{ED} \rightarrow$ Ab given by $\alpha(A, C, \eta)=A$ and $\gamma(A, C, \eta)=C$.

(b) An extended $\eta$-diagram (EED) is a diagram of the form

$$
B \stackrel{\psi}{\rightarrow} A \stackrel{\eta}{\rightarrow} C \stackrel{\chi}{\rightarrow} B
$$

such that $2 \eta=0$ and $\psi \chi=0$ and $\chi \eta \psi=2.1_{B}$. We write EED for the category of extended $\eta$ diagrams. There are evident projection functors $\alpha, \beta, \gamma: \mathrm{EED} \rightarrow \mathrm{Ab}$ as well as a forgetful functor $\pi: \mathrm{EED} \rightarrow \mathrm{ED}$.

Lemma 3.2. Let $(A, B, C, \eta, \chi, \psi)$ be an extended $\eta$-diagram. Then $2 \psi=0$ and $2 \chi=0$ and $4.1_{B}=0$. Thus, $\psi$ and $\chi$ induce maps

$$
C / 2 \stackrel{\chi}{\rightarrow} B \stackrel{\psi}{\rightarrow} A[2]
$$

whose composite is zero.

Proof. We have $2 \psi=\psi \circ\left(2.1_{B}\right)=\psi \chi \eta \psi$, which is zero because $\psi \chi=0$. Similarly we have $2 \chi=\left(2.1_{B}\right) \chi=$ $\chi \eta \psi \chi=0$ and $4.1_{B}=\left(2.1_{B}\right)^{2}=\chi \eta \psi \chi \eta \psi=0$. It follows that $\chi(2 C)=(2 \chi)(C)=0$, so $\chi$ factors through $C / 2$. Similarly $2 \psi(B)=0$, so $\psi(B) \leq A[2]$.

Definition 3.3. An extended $\eta$-diagram is exact if the sequence

$$
C \stackrel{2}{\rightarrow} C \stackrel{\chi}{\rightarrow} B \stackrel{\psi}{\rightarrow} A \stackrel{2}{\rightarrow} A
$$

is exact, or equivalently the associated sequence

$$
C / 2 \stackrel{\chi}{\rightarrow} B \stackrel{\psi}{\rightarrow} A[2]
$$

is short exact. We write EEED for the full subcategory of exact extended $\eta$-diagrams (EEEDs).

Example 3.4. Let $B$ be any free module over $\mathbb{Z} / 4$. We then have an EEED as follows:

$$
B \longrightarrow B / 2 \stackrel{1}{\longrightarrow} B / 2 \stackrel{2}{\longrightarrow} B \text {. }
$$


Lemma 3.5. Let $(A, B, C, \eta, \chi, \psi)$ be an EEED, and let $\bar{\eta}$ denote the composite

$$
A[2] \longleftrightarrow A \stackrel{\eta}{\longrightarrow} C \longrightarrow C / 2 \text {. }
$$

Then $\Phi$ of the extension $C / 2 \rightarrow B \rightarrow A[2]$ is $\bar{\eta}$, so we have natural exact sequences

$$
C / 2 \stackrel{\chi}{\longrightarrow} B[2] \stackrel{\psi}{\longrightarrow} A[2] \stackrel{\bar{\eta}}{\longrightarrow} C / 2 \stackrel{\chi}{\longrightarrow} B / 2 \stackrel{\psi}{\longrightarrow} A[2]
$$

Proof. Consider an element $a \in A[2]$. Choose an element $b \in B$ with $\psi(b)=a$. To determine $\phi(E)(a)$, we must find $\bar{c} \in C / 2$ with $\chi(\bar{c})=2 b$. As $2.1_{B}=\chi \eta \psi$, we can take $\bar{c}=\eta \psi(b)=\eta(a)$. This shows that $\phi(E)=\bar{\eta}$ as claimed, and the rest follows from Proposition 2.1.

Definition 3.6. Let $N=(A, B, C, \eta, \chi, \psi)$ and $N^{\prime}=\left(A^{\prime}, B^{\prime}, C^{\prime}, \eta^{\prime}, \chi^{\prime}, \psi^{\prime}\right)$ be extended $\eta$-diagrams. We define $\xi: \operatorname{Hom}\left(A[2], C^{\prime} / 2\right) \rightarrow \operatorname{EEED}\left(N, N^{\prime}\right)$ as follows. For any map $u: A[2] \rightarrow C^{\prime} / 2$ we let $\bar{u}$ denote the composite

$$
B \stackrel{\psi}{\rightarrow} A[2] \stackrel{u}{\rightarrow} C^{\prime} / 2 \stackrel{\chi^{\prime}}{\rightarrow} B^{\prime},
$$

and note that $\psi^{\prime} \bar{u}=0$ and $\bar{u} \chi=0$. We then let $\xi(u)$ be the morphism $N \rightarrow N^{\prime}$ given by the following diagram:

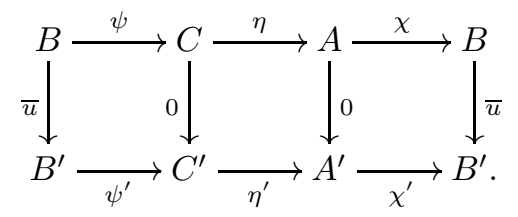

Proposition 3.7. The functor $\pi:$ EEED $\rightarrow$ ED is essentially surjective and reflects isomorphisms. Moreover, for any $N, N^{\prime} \in$ EEED there is a natural short exact sequence

$$
\operatorname{Hom}\left(\alpha(N)[2], \gamma\left(N^{\prime}\right) / 2\right) \stackrel{\xi}{\longrightarrow} \operatorname{EEED}\left(N, N^{\prime}\right) \stackrel{\pi}{\longrightarrow} \operatorname{ED}\left(\pi(N), \pi\left(N^{\prime}\right)\right) .
$$

(In particular, the functor $\pi$ is full.)

Proof. First consider an $\eta$-diagram $P=(A \stackrel{\eta}{\rightarrow} C)$. Let $\bar{\eta}$ denote the composite

$$
A[2] \rightarrow A \stackrel{\eta}{\rightarrow} C \rightarrow C / 2 .
$$

By Proposition 2.1 we can choose an extension $E=(C / 2 \stackrel{\bar{x}}{\rightarrow} B \stackrel{\bar{\psi}}{\rightarrow} A[2])$ with $\Phi(E)=\bar{\eta}$. We define $\chi$ to be the composite $C \rightarrow C / 2 \stackrel{\bar{\chi}}{\rightarrow} B$, and $\psi$ to be the composite $B \stackrel{\bar{\psi}}{\rightarrow} A[2] \rightarrow A$. It is then clear that $\psi \chi=0$. From the construction of $\Phi$ we see that $2.1_{B}=\overline{\chi \eta} \bar{\psi}=\chi \eta \psi$. We thus have an EEED $N=(A, B, C, \eta, \chi, \psi)$ with $\pi(N)=P$, showing that $\pi$ is essentially surjective.

Next, suppose we have another EEED $N^{\prime}=\left(A^{\prime}, B^{\prime}, C^{\prime}, \eta^{\prime}, \chi^{\prime}, \psi^{\prime}\right)$. A morphism from $N$ to $N^{\prime}$ is then a commutative diagram as shown on the left below, which automatically gives rise to a another diagram as shown on the right:
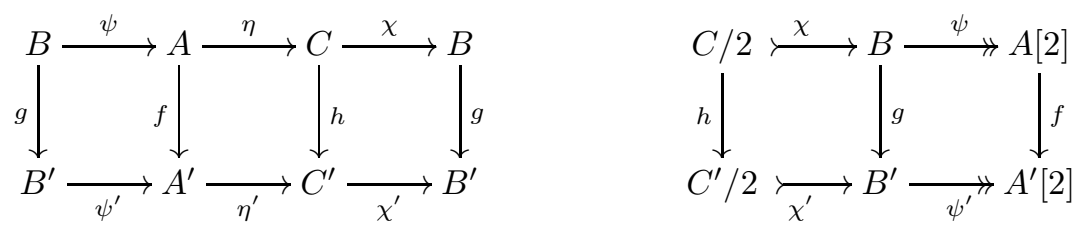

If $f$ and $h$ are isomorphisms, then so are the induced maps $A[2] \rightarrow A^{\prime}[2]$ and $C / 2 \rightarrow C^{\prime} / 2$, so $g$ is also an isomorphism by the five-lemma. This shows that $\pi$ reflects isomorphisms. Suppose instead that $f=0$ and $h=0$. We then have $g \chi=\chi^{\prime} h=0$ and $\psi^{\prime} g=f \psi=0$, so $g$ factors through the cokernel of $\chi$ and the kernel of $\psi^{\prime}$. In other words, there is a unique map $\bar{g}: A[2] \rightarrow C^{\prime} / 2$ such that

$$
g=\left(B \stackrel{\psi}{\rightarrow} A[2] \stackrel{\bar{g}}{\rightarrow} C^{\prime} / 2 \stackrel{\chi^{\prime}}{\rightarrow} B^{\prime}\right),
$$

so $(f, g, h)=\xi(\bar{g})$. This shows that our sequence is left exact. 
Finally, suppose we are given $f$ and $h$ making the middle square on the left commute, but not $g$. Let $E$ and $E^{\prime}$ denote the extensions $C / 2 \rightarrow B \rightarrow A[2]$ and $C^{\prime} / 2 \rightarrow B^{\prime} \rightarrow A^{\prime}[2]$, so $\Phi(E)=\bar{\eta}$ and $\Phi\left(E^{\prime}\right)=\bar{\eta}^{\prime}$ by Lemma 3.5. The relation $h \eta=\eta^{\prime} f$ implies that $h_{*} \Phi(E)=f^{*} \Phi\left(E^{\prime}\right)$, so Proposition 2.3 tells us that there exists $g: B \rightarrow B^{\prime}$ making the right hand diagram commute. It follows that the remaining squares on the left commute also, so we have a morphism $(f, g, h) \in \operatorname{EEED}\left(N, N^{\prime}\right)$ with $\pi(f, g, h)=(f, h)$ as required.

The following result proves is evidence that the work done in this paper is really necessary, and cannot be simplified away.

Proposition 3.8. There is no functor $\sigma:$ ED $\rightarrow$ EEED (additive or otherwise) such that the composite $\pi \sigma: \mathrm{ED} \rightarrow \mathrm{ED}$ is naturally isomorphic to the identity functor.

It will be convenient to give the core of the argument as a separate lemma.

Lemma 3.9. Let ElAb denote the category of elementary abelian 2-groups, and suppose we have a (not necessarily additive) functor $F: \mathrm{ElAb} \rightarrow \mathrm{Ab}$ with a natural short exact sequence $U \stackrel{\chi}{\rightarrow} F(U) \stackrel{\psi}{\rightarrow} U$. Then the sequence is naturally split, so $2 F(U)=0$.

Proof. First, the exact sequence shows that $F(0)=0$. Next, we can choose $x \in F(\mathbb{Z} / 2)$ with $\psi(x)=1$. For an arbitrary group $U \in \mathrm{ElAb}$ and $u \in U$ we have $\alpha_{u}: \mathbb{Z} / 2 \rightarrow U$ with $\alpha_{u}(1)=u$, and we put $\theta(u)=$ $F\left(\alpha_{u}\right)(x) \in F(U)$. This defines a natural function (not obviously a homomorphism) $\theta: U \rightarrow F(U)$ with $\psi \theta=1$. Using $F(0)=0$ we see that $\theta(0)=0$ for all $U$. Now consider the element $\omega_{U}=\sum_{u \in U} \theta(u) \in F(U)$, which is evidently invariant under $\operatorname{Aut}(U)$, so $\psi\left(\omega_{U}\right) \in U^{\operatorname{Aut}(U)}$. Now suppose that $|U|>2$, in which case it is easy to see that $U^{\operatorname{Aut}(U)}=0$. We thus have $\psi\left(\omega_{U}\right)=0$, so $\omega_{U}=\chi\left(\omega_{U}^{\prime}\right)$ for a unique element $\omega_{U}^{\prime} \in U$. This is again invariant under $\operatorname{Aut}(U)$ and so is zero, which implies that $\omega_{U}=0$. Now specialise to the case $U=(\mathbb{Z} / 2)^{2}=\left\{0, e_{1}, e_{2}, e_{1}+e_{2}\right\}$; the conclusion is that $\theta\left(e_{1}\right)+\theta\left(e_{2}\right)+\theta\left(e_{1}+e_{2}\right)=0$. Now define $\alpha_{u, v}:(\mathbb{Z} / 2)^{2} \rightarrow U$ by $\alpha_{u, v}(i, j)=i u+j v$. By applying naturality to this we get $\theta(u)+\theta(v)+\theta(u+v)=0$. We can specialise to the case $u=v$ to get $2 \theta(u)=0$, and then return to the general case to get $\theta(u+v)=$ $\theta(u)+\theta(v)$. Thus, $\theta$ is a natural homomorphism that splits the exact sequence.

Remark 3.10. In the statement of the above lemma we have emphasised that $F$ is not assumed to be additive, because that is necessary for our application. From the conclusion of the lemma we see that $F$ is actually forced to be additive. More generally, as the referee remarked, one can check that any extension of additive functors is automatically additive.

Proof of Proposition 3.8. Suppose we have a functor $\sigma:$ ED $\rightarrow$ EEED, and a natural isomorphism $\lambda: 1 \rightarrow$ $\pi \sigma$. Thus, for each object $L=(U \stackrel{\zeta}{\rightarrow} V) \in$ ED we have an object $\sigma(L)=(B \stackrel{\psi}{\rightarrow} A \stackrel{\eta}{\rightarrow} C \stackrel{\chi}{\rightarrow} B) \in$ EEED together with a commutative diagram

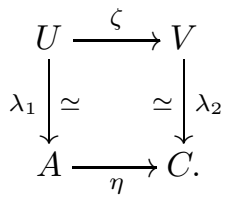

We can define a new functor $\sigma^{\prime}:$ ED $\rightarrow$ EEED by

$$
\sigma^{\prime}(L)=\left(B \stackrel{\lambda_{1}^{-1} \psi}{\longrightarrow} U \stackrel{\zeta}{\rightarrow} V \stackrel{\chi \lambda_{2}}{\longrightarrow} B\right)
$$

and we find that $\sigma^{\prime} \simeq \sigma$ and $\pi \sigma^{\prime}$ is equal (not just isomorphic) to the identity. We will replace $\sigma$ by $\sigma^{\prime}$ and assume that $\pi \sigma=1$. We now have a functor $F: \mathrm{ElAb} \rightarrow$ Ab given by $F(U)=\beta(\sigma(U \stackrel{1}{\rightarrow} U))$, and a natural short exact sequence $U \stackrel{\chi}{\rightarrow} F(U) \stackrel{\psi}{\rightarrow} U$ with $2.1_{F(U)}=\chi \psi$. By the Lemma, this must split, so $2.1_{F(U)}=0$, so $\chi \psi=0$. On the other hand, the sequence is short exact, so $\chi$ is injective and $\psi$ is surjective. This clearly gives a contradiction whenever $U \neq 0$.

We now discuss a slightly different way to think about the category EED. 
Definition 3.11. We define a small additive category $\mathcal{J}$ as follows. There are three objects, denoted by $a$, $b$ and $c$. There are morphisms

$$
b \stackrel{\rho}{\leftarrow} a \stackrel{\eta}{\leftarrow} c \stackrel{\beta}{\leftarrow} b
$$

with $2 \rho=0,2 \eta=0$ and $2 \beta=0$. The full list of morphisms is as follows:

$$
\begin{aligned}
& \mathcal{J}(a, a)=\mathbb{Z} \\
& \mathcal{J}(a, b)=(\mathbb{Z} / 2) \rho \\
& \mathcal{J}(a, c)=0 \\
& \mathcal{J}(b, a)=(\mathbb{Z} / 2) \eta \beta \\
& \mathcal{J}(b, b)=\mathbb{Z} / 4 \\
& \mathcal{J}(c, a)=(\mathbb{Z} / 2) \eta \\
& \mathcal{J}(c, b)=(\mathbb{Z} / 2) \rho \eta \\
& \mathcal{J}(b, c)=(\mathbb{Z} / 2) \beta \\
& \mathcal{J}(c, c)=\mathbb{Z} \text {. }
\end{aligned}
$$

The composition is determined by the rules $\beta \rho=0$ and $2.1_{b}=\rho \eta \beta$.

Proposition 3.12. Any EED $N$ gives an additive functor $Q(N): \mathcal{J}^{o p} \rightarrow$ Ab by the rules

$$
\begin{array}{lll}
Q(N)(a)=A & Q(N)(b)=B & Q(N)(c)=C \\
Q(N)(\beta)=\chi & Q(N)(\eta)=\eta & Q(N)(\rho)=\psi,
\end{array}
$$

so

$$
Q(N)(b \stackrel{\rho}{\leftarrow} a \stackrel{\eta}{\leftarrow} c \stackrel{\beta}{\leftarrow} b)=(B \stackrel{\psi}{\rightarrow} A \stackrel{\eta}{\rightarrow} C \stackrel{\chi}{\rightarrow} B) .
$$

Moreover, this construction gives an equivalence from $\mathrm{EED}$ to the category $\left[\mathcal{J}^{o p}, \mathrm{Ab}\right]$ of additive contravariant functors from $\mathcal{J}$ to $\mathrm{Ab}$.

Proof. Straightforward comparison of definitions.

From now on we will not distinguish notationally between $N$ and $Q(N)$.

Corollary 3.13. For any $x \in \mathcal{J}$ we have a representable functor $F_{x}=\mathcal{J}(-, x): \mathcal{J}^{o p} \rightarrow \mathrm{Ab}$, so $F_{x} \in \mathrm{EED}$. For any morphism $u: x \rightarrow y$ in $\mathcal{J}$ we have a morphism $F_{u}: F_{x} \rightarrow F_{y}$ in EED. The object $F_{x}$ can be displayed as follows:

$$
\begin{aligned}
& F_{b}=(\mathbb{Z} / 4 \longrightarrow \mathbb{Z} / 2 \stackrel{1}{\longrightarrow} \mathbb{Z} / 2 \stackrel{2}{\longrightarrow} \mathbb{Z} / 4) \\
& F_{c}=(\mathbb{Z} / 2 \longrightarrow 0 \longrightarrow \mathbb{Z} \longrightarrow \mathbb{Z} / 2) \\
& F_{a}=(\mathbb{Z} / 2 \longrightarrow \mathbb{Z} \longrightarrow \mathbb{Z} / 2 \longrightarrow \mathbb{Z} / 2) .
\end{aligned}
$$

The morphisms $F_{u}$ are given by the following square. The rows are $F_{b}, F_{c}, F_{a}$ and $F_{b}$ respectively. The maps from the first row to the second comprise the morphism $F_{\beta}: F_{b} \rightarrow F_{a}$. Similarly, the second group of vertical maps comprise the morphism $F_{\eta}: F_{c} \rightarrow F_{a}$, and the third group comprise $F_{\rho}: F_{a} \rightarrow F_{b}$.

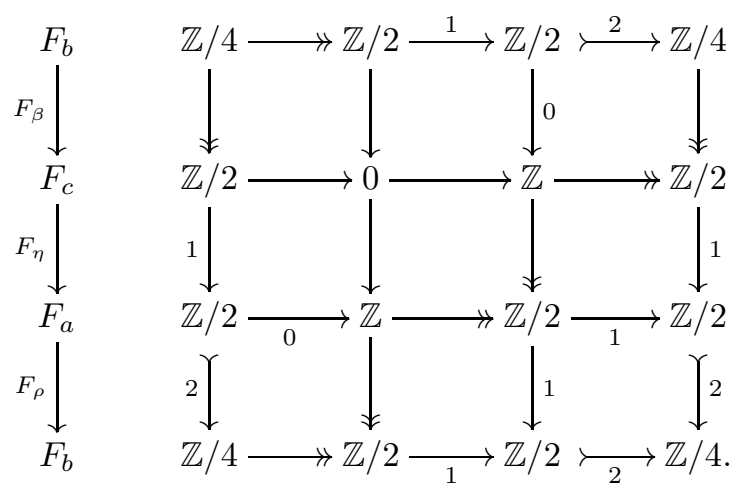

By the Yoneda Lemma we have $\operatorname{EED}\left(F_{x}, N\right)=N(x)$. 
Proof. This is a straightforward (if somewhat lengthy) calculation from the definitions. For example, the first row is

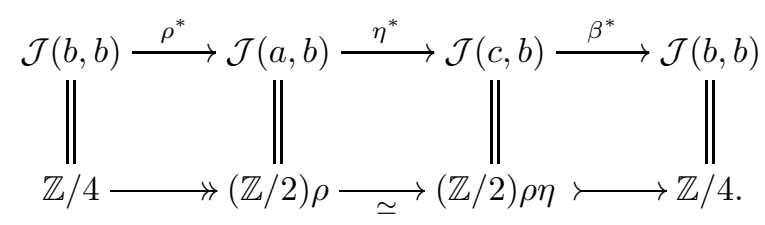

Definition 3.14. Given subcategories $\mathcal{A}, \mathcal{C} \subseteq \mathrm{Ab}$ we write $\operatorname{ED}[\mathcal{A}, \mathcal{C}]$ for the subcategory of $\mathrm{ED}$ consisting of $\eta$-diagrams $A \stackrel{\eta}{\rightarrow} C$ with $A \in \mathcal{A}$ and $C \in \mathcal{C}$. We will particularly be interested in the categories

$$
\begin{aligned}
& \mathcal{F}=\left\{A \in \mathrm{Ab} \mid 2.1_{A} \text { is injective }\right\}=\{A \mid A[2]=0\} \\
& \mathcal{D}=\left\{C \in \mathrm{Ab} \mid 2.1_{C} \text { is surjective }\right\}=\{C \mid C / 2=0\} .
\end{aligned}
$$

Remark 3.15. Consider an exact extended $\eta$-diagram $M=(A, B, C, \eta, \chi, \psi)$, so we have a short exact sequence $C / 2 \stackrel{\chi}{\rightarrow} B \stackrel{\psi}{\rightarrow} A[2]$. If $\chi=0$ we must have $C / 2=0$ (so $C \in \mathcal{D}$ ) and $\psi$ gives an isomorphism $B \rightarrow A[2]$. Similarly, if $\psi=0$ then $A \in \mathcal{F}$ and $C / 2 \simeq B$. Arguing along these lines, we find that:

- The functor $\pi$ gives an equivalence $\{M \mid \chi=0\} \rightarrow \operatorname{ED}[\mathrm{Ab}, \mathcal{D}]$.

Moreover, $\alpha$ gives an equivalence $\{M \mid C=0\} \rightarrow \mathrm{Ab}$.

- The functor $\pi$ gives an equivalence $\{M \mid \psi=0\} \rightarrow \operatorname{ED}[\mathcal{F}, \mathrm{Ab}]$.

Moreover, $\gamma$ gives an equivalence $\{M \mid A=0\} \rightarrow \mathrm{Ab}$.

- The functor $\pi$ also gives an equivalence $\{M \mid B=0\} \rightarrow \operatorname{ED}[\mathcal{F}, \mathcal{D}]$.

To complete the picture, we should analyse the case where $\eta=0$. This is a little more elaborate.

Definition 3.16. We define a category SPP (short for "split Postnikov pairs") as follows. The objects are pairs $(A, C)$ of abelian groups. The morphisms from $\left(A_{0}, C_{0}\right)$ to $\left(A_{1}, C_{1}\right)$ are triples $(f, g, u)$ where $f: A_{0} \rightarrow A_{1}$ and $h: C_{0} \rightarrow C_{1}$ and $u: A_{0}[2] \rightarrow C_{1} / 2$. The identity morphism of $(A, C)$ is $\left(1_{A}, 1_{C}, 0\right)$. The composite of

$$
\left(A_{0}, C_{0}\right) \stackrel{\left(f_{0}, h_{0}, u_{0}\right)}{\longrightarrow}\left(A_{1}, C_{1}\right) \stackrel{\left(f_{1}, h_{1}, u_{1}\right)}{\longrightarrow}\left(A_{2}, C_{2}\right)
$$

is $\left(f_{1} f_{0}, h_{1} h_{0},\left(h_{1}\right)_{*} u_{0}+\left(f_{0}\right)^{*} u_{1}\right)$. (We leave it to the reader to check that this is associative and unital.) Next, for $(A, C) \in \mathrm{SPP}$ we define $H(A, C) \in$ EEED to be the diagram

$$
C / 2 \oplus A[2] \stackrel{\psi}{\rightarrow} A \stackrel{0}{\rightarrow} C \stackrel{\chi}{\rightarrow} C / 2 \oplus A[2]
$$

where $\psi(\bar{c}, a)=a$ and $\chi(c)=(c+2 C, 0)$. Given a morphism $(f, h, u):\left(A_{0}, C_{0}\right) \rightarrow\left(A_{1}, C_{1}\right)$ we define $g: C_{0} / 2 \oplus A_{0}[2] \rightarrow C_{1} / 2 \oplus A_{1}[2]$ by

$$
g\left(c_{0}+2 C_{0}, a_{0}\right)=\left(h\left(c_{0}\right)+u\left(a_{0}\right)+2 C_{1}, f\left(a_{0}\right)\right) .
$$

We find that the diagram

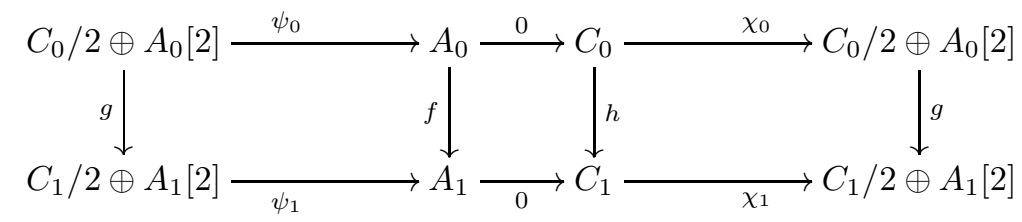

commutes, so we have a morphism $(f, g, h) \in \operatorname{EEED}\left(H\left(A_{0}, C_{0}\right), H\left(A_{1}, C_{1}\right)\right)$. We write $H(f, h, u)$ for this morphism.

Proposition 3.17. The above construction gives an equivalence $H: \operatorname{SPP} \rightarrow\{M \in \operatorname{EEED} \mid \eta=0\}$.

Proof. We leave it to the reader to check that $H$ gives an isomorphism

$$
\operatorname{SPP}\left(\left(A_{0}, C_{0}\right),\left(A_{1}, C_{1}\right)\right) \rightarrow \operatorname{EEED}\left(H\left(A_{0}, C_{0}\right), H\left(A_{1}, C_{1}\right)\right),
$$

and that this is compatible with composition. Thus, $H$ defines a full and faithful embedding SPP $\rightarrow$ EEED. Now consider an EEED $M$ with $\eta=0$, say $M=(A, B, C, 0, \chi, \psi)$. We then have $2.1_{B}=\psi \eta \chi=0$, so 
the sequence $C / 2 \rightarrow B \rightarrow A[2]$ is a short exact sequence of vector spaces over the field $\mathbb{Z} / 2$, so it splits. Any choice of splitting gives an isomorphism $H(A, C) \rightarrow M$. It follows that the essential image of $H$ is $\{M \mid \eta=0\}$, as claimed.

We now explain the precise connection between Moore diagrams and $\eta$-diagrams.

Proposition 3.18. Let EMD' denote the full subcategory of EEED consisting of diagrams $(A, B, C, \eta, \chi, \psi)$ for which the map $\eta: A \rightarrow C$ is surjective with kernel $2 A$. Then there is an equivalence $E$ : EMD $\rightarrow$ EMD $^{\prime}$ given by

$$
E(B \stackrel{\psi}{\rightarrow} A \stackrel{\phi}{\rightarrow} B)=(B \stackrel{\psi}{\rightarrow} A \stackrel{\text { proj }}{\rightarrow} A / 2 \stackrel{\Phi}{\rightarrow} B)
$$

(where $\bar{\phi}$ is the unique map with $\bar{\phi} \circ \operatorname{proj}=\phi$ ).

Proof. The inverse functor is just

$$
E^{-1}(B \stackrel{\psi}{\rightarrow} A \stackrel{\eta}{\rightarrow} C \stackrel{\chi}{\rightarrow} B)=(B \stackrel{\psi}{\rightarrow} A \stackrel{\chi \eta}{\rightarrow} B) .
$$

We leave all further details to the reader.

Lemma 3.19. If $N \in \mathrm{EMD}^{\prime}$ and $N^{\prime} \in \mathrm{EEED}$ then the map $\alpha: \operatorname{ED}\left(\pi(N), \pi\left(N^{\prime}\right)\right) \rightarrow \operatorname{Hom}\left(\alpha(N), \alpha\left(N^{\prime}\right)\right)$ is a bijection.

Proof. If we write $N$ and $N^{\prime}$ in the usual form, then $\operatorname{ED}\left(\pi(N), \pi\left(N^{\prime}\right)\right)$ is the set of pairs $(f, h)$ making the following diagram commute:

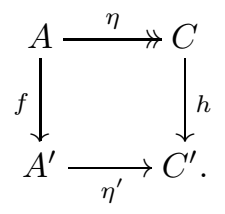

If we are given an arbitrary homorphism $f: A \rightarrow A^{\prime}$, we can define $h: C \rightarrow C^{\prime}$ as follows. Given $a \in A$, we choose $c \in C$ with $\eta(c)=a$, then put $h(a)=\eta^{\prime}(f(c))$. By assumption we have $\operatorname{ker}(\eta)=2 A$ and $2 \eta^{\prime}=0$ so $\eta^{\prime}(f(2 A))=0$, which proves that $h$ is well-defined. It is clear that $h$ is the unique homomorphism making the diagram commute. Thus, the projection map $(f, h) \mapsto f$ is an isomorphism as claimed.

Corollary 3.20. The functor $\alpha: \mathrm{EMD} \rightarrow \mathrm{Ab}$ is full and essentially surjective, and reflects isomorphisms. For any $M, M^{\prime} \in \mathrm{EMD}$ there is a natural short exact sequence

$$
\operatorname{Hom}\left(\alpha(M)[2], \alpha\left(M^{\prime}\right) / 2\right) \rightarrow \operatorname{EMD}\left(M, M^{\prime}\right) \rightarrow \operatorname{Hom}\left(\alpha(M), \alpha\left(M^{\prime}\right)\right) .
$$

Proof. Put $N=E(M)$ and $N^{\prime}=E\left(M^{\prime}\right)$, so these are objects in the full subcategory EMD $\subseteq$ EEED. Proposition 3.7 gives a short exact sequence

$$
\operatorname{Hom}\left(\alpha(N)[2], \gamma\left(N^{\prime}\right) / 2\right) \stackrel{\xi}{\longrightarrow} \operatorname{EEED}\left(N, N^{\prime}\right) \stackrel{\pi}{\longrightarrow} \operatorname{ED}\left(\pi(N), \pi\left(N^{\prime}\right)\right) .
$$

From the definition of $E$ we have $\alpha(N)=\alpha(M)$ and $\gamma\left(N^{\prime}\right) / 2=\alpha\left(M^{\prime}\right) / 2$. Proposition 3.18 identifies $\operatorname{EEED}\left(N, N^{\prime}\right)$ with $\operatorname{EMD}\left(M, M^{\prime}\right)$, and Lemma 3.19 identifies $\operatorname{ED}\left(\pi(N), \pi\left(N^{\prime}\right)\right)$ with $\operatorname{Hom}\left(\alpha(M), \alpha\left(M^{\prime}\right)\right)$. The above short exact sequence can thus be rewritten as

$$
\operatorname{Hom}\left(\alpha(M)[2], \alpha\left(M^{\prime}\right) / 2\right) \rightarrow \operatorname{EMD}\left(M, M^{\prime}\right) \rightarrow \operatorname{Hom}\left(\alpha(M), \alpha\left(M^{\prime}\right)\right),
$$

as claimed. This shows that $\alpha: \mathrm{EMD} \rightarrow \mathrm{Ab}$ is full.

We could prove that $\alpha$ is essentially surjective by a similar process of translation. More directly, for any abelian group $A$ we can take $B=A / 2 \oplus A[2]$ and $\phi(a)=(a+2 A, 0)$ and $\psi\left(a+2 A, a^{\prime}\right)=a^{\prime}$; this gives an exact Moore diagram $M=(A, B, \phi, \psi)$ with $\alpha(M)=A$, proving that $\alpha$ is essentially surjective.

Similarly, suppose we have a morphism $p: M \rightarrow M^{\prime}$ in EMD, given by maps $f: A \rightarrow A^{\prime}$ and $g: B \rightarrow B^{\prime}$, and suppose that $\alpha(p)=f$ is an isomorphism. We then have a commutative diagram with exact rows as 
follows:

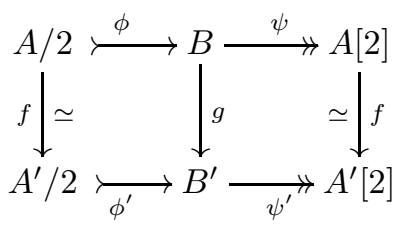

It follows that $g$ is also an isomorphism, so $p$ is an isomorphism in EMD.

\section{Functors from Spectra}

In order to construct extended $\eta$-diagrams from spectra, we need some low-dimensional calculations of stable homotopy groups. In discussing these, it is helpful to remember that the graded groups $[X, Y]_{*}$ are naturally modules over $\pi_{*}(S)$, and composition is bilinear. For example, if we have a map $f: \Sigma^{k} X \rightarrow Y$ then the following composites are all the same and can be denoted by $\eta . f$ :

$$
\left(\Sigma^{k+1} X \stackrel{\eta \wedge 1_{X}}{\longrightarrow} \Sigma^{k} X \stackrel{f}{\rightarrow} Y\right)=\left(\Sigma^{k+1} X \stackrel{\eta \wedge f}{\longrightarrow} Y\right)=\left(\Sigma^{k+1} X \stackrel{\Sigma f}{\longrightarrow} \Sigma Y \stackrel{\eta \wedge 1_{Y}}{\longrightarrow} Y\right) .
$$

(We have suppressed any mention of signs here because $2 \eta=0$.) Similar remarks apply with $\eta$ replaced by $2: S \rightarrow S$. In particular we have the cofibration sequence

$$
S \stackrel{2}{\rightarrow} S \stackrel{\rho}{\rightarrow} S / 2 \stackrel{\beta}{\rightarrow} S^{1} \stackrel{2}{\rightarrow} S^{1}
$$

in which adjacent composites vanish, so $2 . \rho=0$ and $2 . \beta=0$.

Proposition 4.1. The identity map of $S / 2$ has order 4 , and the following diagram commutes:

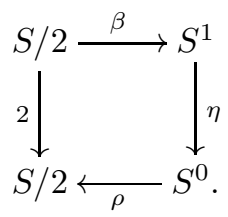

Proof. This is classical, probably due to Toda [5]. One argument is as follows. We have $S / 2=\Sigma^{-1} \mathbb{R} P^{2}$, so $H^{*}(S / 2 ; \mathbb{Z} / 2)=\mathbb{Z} / 2\{a, b\}$ with $|a|=0$ and $|b|=1$ and $\mathrm{Sq}^{1}(a)=b$. This gives

$$
H^{*}(S / 2 \wedge S / 2)=(\mathbb{Z} / 2)\{a \otimes a, a \otimes b, b \otimes a, b \otimes b\}
$$

and by the Cartan formula we have $\operatorname{Sq}^{2}(a \otimes a)=b \otimes b \neq 0$. On the other hand, if we let $\iota$ denote the identity map of $S / 2$, we see that $S / 2 \wedge S / 2$ is the cofibre of $2 \iota$. If $2 \iota$ were zero then $S / 2 \wedge S / 2$ would split as $S / 2 \vee S^{1} / 2$ and we would have $\mathrm{Sq}^{2}=0$ in cohomology, which is false. Thus, we must have $2 \iota \neq 0$. On the other hand, the cofibration sequence $S \stackrel{\rho}{\rightarrow} S / 2 \stackrel{\beta}{\rightarrow} S^{1}$ gives an exact sequence

$$
\pi_{1}(S / 2)=\left[S^{1}, S / 2\right] \stackrel{\beta^{*}}{\longrightarrow}[S / 2, S / 2] \stackrel{\rho^{*}}{\rightarrow}[S, S / 2]=\pi_{0}(S / 2)
$$

We will take it as given that $\pi_{0}(S)=\mathbb{Z}$ and $\pi_{1}(S)=(\mathbb{Z} / 2) \eta$ and $\pi_{2}(S)=(\mathbb{Z} / 2) \eta^{2}$. There is a long exact sequence

$$
\pi_{i}(S) \stackrel{2}{\rightarrow} \pi_{i}(S) \stackrel{\rho_{*}}{\rightarrow} \pi_{i}(S / 2) \stackrel{\beta_{*}}{\rightarrow} \pi_{i-1}(S) \stackrel{2}{\rightarrow} \pi_{i-1}(S) .
$$

From this we read off that $\pi_{0}(S / 2)=(\mathbb{Z} / 2) \rho$ and $\pi_{1}(S / 2)=(\mathbb{Z} / 2) \rho \eta$. Thus, our previous exact sequence looks like

$$
(\mathbb{Z} / 2) \rho \eta \stackrel{\beta^{*}}{\rightarrow}[S / 2, S / 2] \stackrel{\rho^{*}}{\rightarrow}(\mathbb{Z} / 2) \rho,
$$

and of course $\rho^{*}(\iota)=\rho$. The only way we can have $2 \iota \neq 0$ is if $\beta^{*}$ is injective and $2 \iota=\beta^{*}(\rho \eta)=\rho \eta \beta$ as claimed.

Corollary 4.2. There is a full and faithful additive embedding $T: \mathcal{J} \rightarrow$ Spectra given by $T(a)=S$ and $T(b)=S / 2$ and $T(c)=S^{1}$.

Corollary 4.3. For any spectrum $X$ there is an exact extended $\eta$-diagram $G(X): \mathcal{J}^{\text {op }} \rightarrow$ Ab given by $G(X)(x)=[T(x), X]$ for all $x \in \mathcal{J}$. In the notation of Corollary 3.13, we have $G(S)=F_{a}, G(S / 2)=F_{b}$ and $G\left(S^{1}\right)=F_{c}$. 
Proof. It is formal that the given rule defines an additive functor $\mathcal{J}^{\text {op }} \rightarrow \mathrm{Ab}$, or in other words an extended $\eta$ diagram. The sequence

$$
T(a) \stackrel{2}{\rightarrow} T(a) \stackrel{T(\rho)}{\longrightarrow} T(b) \stackrel{T(\beta)}{\longrightarrow} T(c) \stackrel{2}{\rightarrow} T(c)
$$

is the cofibration sequence

$$
S \stackrel{2}{\rightarrow} S \stackrel{\rho}{\rightarrow} S / 2 \stackrel{\beta}{\rightarrow} S^{1} \stackrel{2}{\rightarrow} S^{1}
$$

so when we apply $[-, X]$ we get an exact sequence

$$
G(X)(a) \stackrel{2}{\leftarrow} G(X)(a) \stackrel{\psi}{\leftarrow} G(X)(b) \stackrel{\chi}{\leftarrow} G(X)(c) \stackrel{2}{\leftarrow} G(X)(c)
$$

or equivalently

$$
G(X)(c) / 2 \stackrel{\chi}{\longrightarrow} G(X)(b) \stackrel{\psi}{\longrightarrow} G(X)(a)[2] .
$$

Thus, we actually have an exact extended $\eta$-diagram. As $T$ is full and faithful we have

$$
G(T(w))(x)=[T(x), T(w)]=\mathcal{J}(x, w)=F_{w}(x),
$$

so $G(T(w))=F_{w}$. This gives $G(S)=F_{a}, G(S / 2)=F_{b}$ and $G\left(S^{1}\right)=F_{c}$ as claimed.

Remark 4.4. We will prove as Corollary 5.13 that $G$ is essentially surjective. Lemma 4.13 describes a subcategory on which it reflects isomorphism. Corollary 5.12 gives a smaller subcategory where $G$ is full, together with a good description of the kernel of the map $G:[X, Y] \rightarrow \operatorname{EEED}(G(X), G(Y))$ in that context.

Example 4.5. Given any pair of abelian groups $A$ and $C$, we have Eilenberg-MacLane spectra $H A$ and $H C$ and we find that $G(H A \vee \Sigma H C)=G(H A) \oplus G(\Sigma H C)$ is the diagram $H(A, C)$ defined in Definition 3.16 However, the resulting map

$$
\left[H A_{0} \vee \Sigma H C_{0}, H A_{1} \vee \Sigma H C_{1}\right] \rightarrow \operatorname{SPP}\left(\left(A_{0}, C_{0}\right),\left(A_{1}, C_{1}\right)\right)=\operatorname{EEED}\left(H\left(A_{0}, C_{0}\right), H\left(A_{1}, C_{1}\right)\right)
$$

is not an isomorphism in general. To cure this, let $\mathrm{SPP}^{+}$be the category with the same objects as $\mathrm{SPP}$, and morphisms

$$
\operatorname{SPP}^{+}\left(\left(A_{0}, C_{0}\right),\left(A_{1}, C_{1}\right)\right)=\operatorname{Hom}\left(A_{0}, A_{1}\right) \times \operatorname{Hom}\left(C_{0}, C_{1}\right) \times \operatorname{Ext}\left(A_{0}, C_{1}\right),
$$

with composition

$$
\left(f_{1}, g_{1}, u_{1}\right) \circ\left(f_{0}, g_{0}, u_{0}\right)=\left(f_{1} f_{0}, g_{1} g_{0},\left(g_{1}\right)_{*}\left(u_{0}\right)+f_{0}^{*}\left(u_{1}\right)\right) .
$$

Using the Hurewicz and Universal Coefficient theorems, we obtain natural isomorphisms

$$
\begin{aligned}
{\left[H A_{0}, H A_{1}\right] } & \simeq \operatorname{Hom}\left(A_{0}, A_{1}\right) & {\left[\Sigma H C_{0}, H A_{1}\right] } & \simeq 0 \\
{\left[H A_{0}, \Sigma H C_{1}\right] } & \simeq \operatorname{Ext}\left(A_{0}, C_{1}\right) & {\left[\Sigma H C_{0}, \Sigma H C_{1}\right] } & \simeq \operatorname{Hom}\left(C_{0}, C_{1}\right) .
\end{aligned}
$$

Using this we see that there is a full and faithful embedding $\widetilde{H}: \operatorname{SPP}^{+} \rightarrow$ Spectra given by $\widetilde{H}(A, C)=$ $H A \vee \Sigma H C$. The map $\Phi: \operatorname{Ext}\left(A_{0}, C_{1}\right) \rightarrow \operatorname{Hom}\left(A_{0}[2], C_{1} / 2\right)$ from Proposition 2.1 gives rise to a functor $\mathrm{SPP}^{+} \rightarrow \mathrm{SPP}$, which we also denote by $\Phi$. We then find that the following diagram commutes up to natural isomorphism:

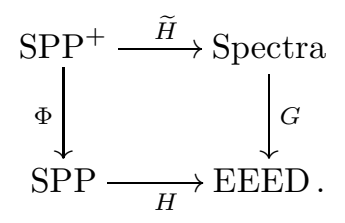

Definition 4.6. For any spectra $X$ and $Y$, we define

$$
\begin{aligned}
L(X, Y) & =\operatorname{ker}(G:[X, Y] \rightarrow \operatorname{EEED}(G(X), G(Y))) \\
K(X, Y) & =\operatorname{ker}(\pi G:[X, Y] \rightarrow \operatorname{ED}(\pi G(X), \pi G(Y))) \\
& =\left\{f: X \rightarrow Y \mid \pi_{0}(f)=0 \text { and } \pi_{1}(f)=0\right\} .
\end{aligned}
$$

For any $f \in K(X, Y)$ we form a cofibration sequence $X \rightarrow Y \rightarrow C f \rightarrow \Sigma X$, giving an exact sequence

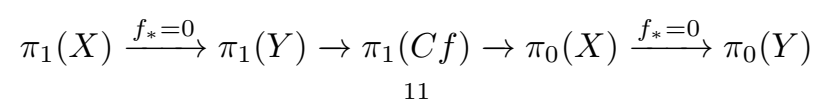


and thus an extension

$$
E(f)=\left(\pi_{1}(Y) \longleftrightarrow \pi_{1}(C f) \longrightarrow \pi_{0}(X)\right) .
$$

Standard results about uniqueness of cofibration sequences show that this is well-defined up to equivalence, we have a well-defined class $\omega(f)=[E(f)] \in \operatorname{Ext}\left(\pi_{0}(X), \pi_{1}(Y)\right)$.

Lemma 4.7. The map $\omega: K(X, Y) \rightarrow \operatorname{Ext}\left(\pi_{0}(X), \pi_{1}(Y)\right)$ is a homomorphism. Moreover, given maps $W \stackrel{p}{\rightarrow} X \stackrel{f}{\rightarrow} Y \stackrel{q}{\rightarrow} Z$ with $f \in K(X, Y)$ we have

$$
\omega(q f p)=\pi_{1}(q)_{*} \pi_{0}(p)^{*} \omega(f) \in \operatorname{Ext}\left(\pi_{0}(W), \pi_{1}(Z)\right),
$$

Proof. First suppose we have $X \stackrel{f}{\rightarrow} Y \stackrel{q}{\rightarrow} Z$ with $f \in K(X, Y)$. The octahedral axiom then gives a commutative diagram

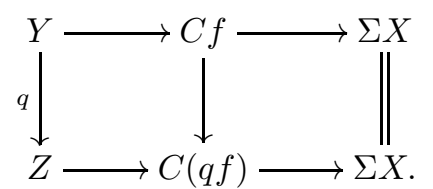

We can apply $\pi_{1}$ to get a morphism of extensions, and then use the first part of Proposition 2.3 to deduce that $\omega(q f)=\pi_{1}(q)_{*} \omega(f)$. A similar argument shows that $\omega(f p)=\pi_{0}(p)^{*} \omega(f)$. Now consider the diagonal map $X \rightarrow X \vee X$ and the codiagonal map $\nabla: Y \vee Y \rightarrow Y$. Given another map $f^{\prime} \in K(X, Y)$ we have

$$
\omega\left(f+f^{\prime}\right)=\omega\left(\nabla \circ\left(f \vee f^{\prime}\right) \circ \Delta\right)=\nabla_{*} \Delta^{*}\left(\omega(f) \oplus \omega\left(f^{\prime}\right)\right)=\omega(f)+\omega\left(f^{\prime}\right),
$$

so $\omega$ is a homomorphism.

Proposition 4.8. There is a natural commutative diagram as follows, in which the rectangle and the squares are pullbacks:

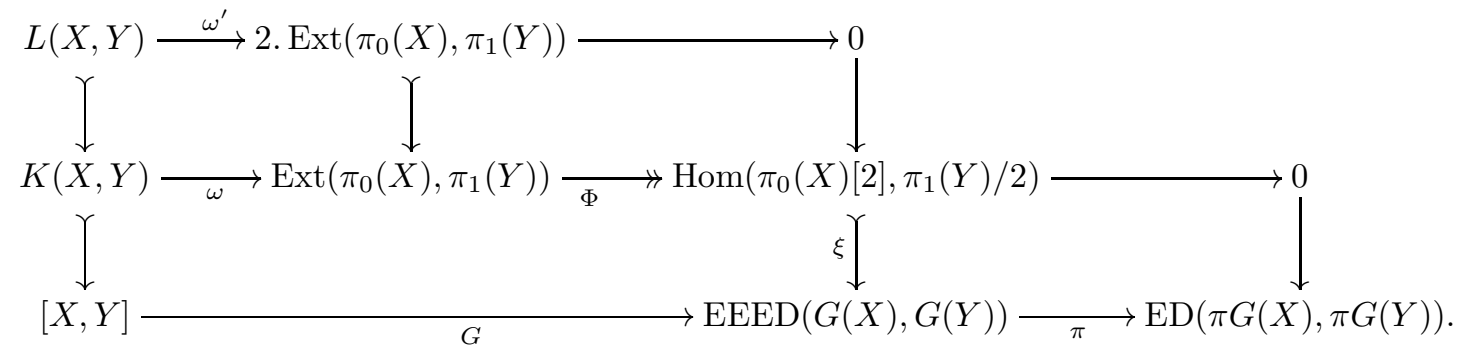

Moreover:

(a) If the maps $\pi G$ and $\omega$ are surjective, then so is $G$.

(b) If $\omega$ is an isomorphism then so is $\omega^{\prime}$.

(c) If $\omega$ is an isomorphism and $2 . \operatorname{Ext}\left(\pi_{0}(X), \pi_{1}(Y)\right)=0$, then $G$ is injective.

Proof. The bottom right square is a pullback (with $\pi$ surjective) by Proposition 3.7, and the top middle square is a pullback (with $\Phi$ surjective) by Proposition 2.1. We next show that the bottom left rectangle commutes. Consider an element $f \in K(X, Y)$, so $\pi G(f)=0$. As $\xi$ is the kernel of $\pi$, we have $G(f)=\xi(s)$ for some $s: \pi_{0}(X)[2] \rightarrow \pi_{1}(Y) / 2$. We need to show that $s=\Phi(\omega(f))$. Consider an element $w \in \pi_{0}(X)[2]$. By inspecting the definitions, we see that $s(w)$ can be described as follows: we choose any $v: S / 2 \rightarrow X$ with $v \rho=w$, then choose any $u \in \pi_{1}(Y)$ with $u \beta=f v: S / 2 \rightarrow Y$, then $s(w)$ is the coset $u+2 \pi_{1}(Y)$. The best way to choose $u$ is as follows. Let $h: \Sigma^{-1} C f \rightarrow X$ be the connecting map. As $\pi_{0}(f)=0$ we have $f w=0$, so we can choose $z: S \rightarrow \Sigma^{-1} C f$ with $h z=w=v \rho$. This gives the solid part of the diagram below, in which the rows are cofibration sequences:

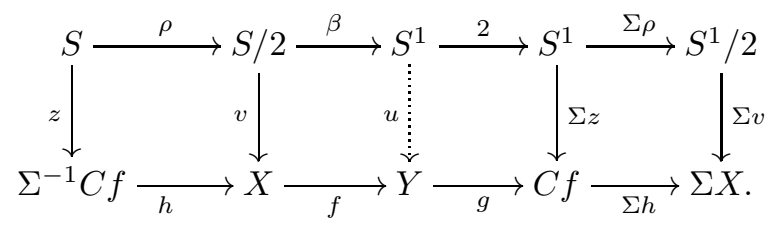

12 
By the axioms for a triangulated category, there is a map $u$ making everything commute. In particular we have $u \beta=f v$ so $u$ represents $s(w)$. On the other hand, we can also apply $\pi_{1}$ to obtain a commutative diagram

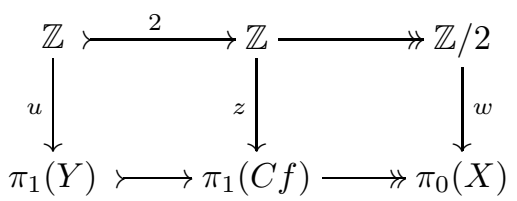

from which we can read off the fact that $\Phi(\omega(f))(w)$ is represented by $u$. Thus $\xi \Phi \omega=\left.G\right|_{K(X, Y)}$ as claimed. Moreover, we have $f \in L(X, Y)$ iff $G(f)=0$ iff $\xi \Phi \omega(f)=0$ iff $\Phi \omega(f)=0$ iff $\omega(f) \in \operatorname{ker}(\Phi)=$ 2 . $\operatorname{Ext}\left(\pi_{0}(X), \pi_{1}(Y)\right)$. We can thus define $\omega^{\prime}$ to be the restriction of $\omega$, and this makes the top left square a pullback. It also follows formally from the definition of $K(X, Y)$ that the bottom left rectangle is a pullback. The auxilary statements (a), (b) and (c) now follow easily by chasing the diagram.

Lemma 4.9. There is a finite spectrum $Q$ fitting in a diagram as follows, in which the rows and columns are cofibration sequences.

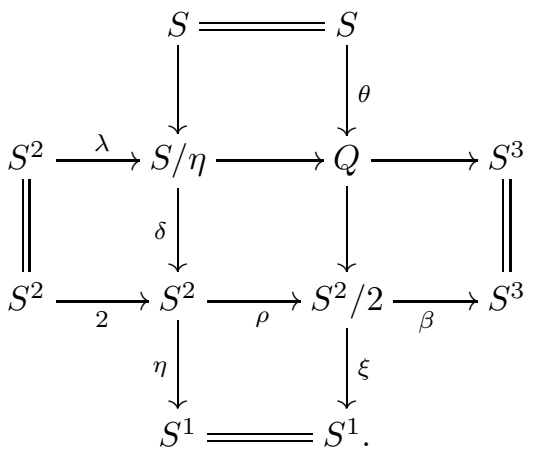

Moreover, the unit map $S \rightarrow H$ factors uniquely through $\theta: S \rightarrow Q$, and the fibre of the resulting map $Q \rightarrow H$ is 2-connected.

This is a fairly well-known example in low-dimensional stable homotopy theory, and is often characterised by the "cell diagram"

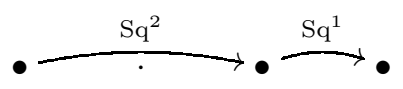

However, it will be simpler to prove the precise properties that we need rather than trying to extract them from the literature.

Proof. The third row is a familiar cofibration sequence. We define $S / \eta$ to be the cofibre of the map $\eta: S^{1} \rightarrow S$. (This can also be described as $\Sigma^{-2} \mathbb{C} P^{2}$.) We let $\delta$ denote the connecting map, so that the second column in the diagram is also a cofibration sequence. Using this cofibration sequence and our knowledge of $\pi_{\leq 2}(S)$, we see that there is a unique element $\lambda \in \pi_{2}(S / 2)$ with $\delta \lambda=2$, so that the left square commutes. We also find that $\pi_{0}(S / \eta)=\mathbb{Z}$ and $\pi_{1}(S / \eta)=0$ and $\pi_{2}(S / \eta)=\mathbb{Z} \lambda$.

Next, we define $Q$ to be the cofibre of $\lambda$, so the second row is another cofibration sequence. The octahedral axiom now tells us that the third column can be filled in as claimed. If we apply the functor $[-, H]$ to that column we see that the unit map $S \rightarrow H$ factors uniquely through $\theta$. We can also apply $\pi_{*}$ to the second row to see that $\pi_{0}(Q)=\mathbb{Z}$ and $\pi_{1}(Q)=\pi_{2}(Q)=0$. Now let $F$ be the fibre of the map $Q \rightarrow H$. We then have $\pi_{0}(F)=0$ and $\pi_{k}(F)=\pi_{k}(Q)$ for $k>0$, so in particular $\pi_{1}(F)=\pi_{2}(F)=0$ as claimed.

Remark 4.10. It is possible to give a more geometric construction, as follows. Let $\mathbb{H}$ denote the space of quaternions, and $\mathbb{H}_{0}$ the subspace of purely imaginary ones. There are evident maps

$$
P_{\mathbb{R}}\left(\mathbb{H} \oplus \mathbb{H}_{0}\right) \rightarrow P_{\mathbb{R}}(\mathbb{H} \oplus \mathbb{H}) \rightarrow P_{\mathbb{H}}(\mathbb{H} \oplus \mathbb{H}) \simeq \mathbb{H}_{\infty} \simeq S^{4} .
$$

The composite sends $P_{\mathbb{R}}\left(0 \oplus \mathbb{H}_{0}\right)$ to the basepoint. Thus, we have an induced map $f$ from the quotient space $P=P_{\mathbb{R}}\left(\mathbb{H} \oplus \mathbb{H}_{0}\right) / P_{\mathbb{R}}\left(0 \oplus \mathbb{H}_{0}\right) \simeq \mathbb{R} P^{6} / \mathbb{R} P^{2}$ to $S^{4}$. We put $Q=\Sigma^{-4} C f$. 
We have $H^{*}(P ; \mathbb{Z} / 2)=(\mathbb{Z} / 2)\left\{a_{3}, a_{4}, a_{5}, a_{6}\right\}$ with $\operatorname{Sq}^{1}\left(a_{3}\right)=a_{4}$ and $\operatorname{Sq}^{1}\left(a_{5}\right)=a_{6}$ and $\operatorname{Sq}^{2}\left(a_{3}\right)=a_{5}$. One can check that $f$ restricts to give a map $P_{\mathbb{R}}\left(\mathbb{C} \oplus \mathbb{H}_{0}\right) / P_{\mathbb{R}}\left(0 \oplus H_{0}\right) \rightarrow S^{4}$ that is generically bijective, and therefore sends the generator of $H^{4}\left(S^{4} ; \mathbb{Z} / 2\right)$ to $a_{4}$. It follows that $H^{*}(Q ; \mathbb{Z} / 2)$ has the form $(\mathbb{Z} / 2)\left\{b_{0}, b_{2}, b_{3}\right\}$ with $\left|b_{i}\right|=i$ and $\mathrm{Sq}^{2}\left(b_{0}\right)=b_{2}$ and $\mathrm{Sq}^{1}\left(b_{2}\right)=b_{3}$. From this one can deduce that $Q$ fits into a system of cofibration sequences as indicated. We leave further details of this approach to the reader.

We next recall a standard fact for ease of reference.

Lemma 4.11. Let $X$ be a connective spectrum.

(a) If $H_{*}(X)=0$ then $X$ is contractible.

(b) If $i$ is the smallest integer such that $H_{i}(X) \neq 0$, then the Hurewicz map $\pi_{i}(X) \rightarrow H_{i}(X)$ is an isomorphism.

Proof. First suppose that $H_{*}(X)=0$. By assumption $X$ is connective, so there exists $n$ such that $\pi_{j}(X)=0$ for all $j<n$. The Hurewicz theorem then tells us that $\pi_{n}(X) \simeq H_{n}(X)=0$. This allows us to apply the Hurewicz theorem one dimension higher, so $\pi_{n+1}(X) \simeq H_{n+1}(X)=0$. We can continue this inductively to see that $\pi_{i}(X)=0$ for all $X$, so $X$ is contractible. The proof for (b) is essentially the same.

Remark 4.12. Connectivity is certainly required here; for example, the spectrum $X=K U \wedge S / 2$ is not contractible but has $H_{*}(X)=0$.

Lemma 4.13. Let $\mathcal{C}$ be the category of $(-1)$-connected spectra $X$ with $H_{k}(X)=0$ for $k>1$. Then for $X \in \mathcal{C}$ there is a natural short exact sequence

$$
\pi_{0}(X) / 2 \stackrel{\eta}{\longrightarrow} \pi_{1}(X) \stackrel{h}{\longrightarrow} H_{1}(X) .
$$

Moreover, the functor $G: \mathcal{C} \rightarrow$ EEED reflects isomorphism.

Proof. Let $Q$ be as in Lemma 4.9, and let $F$ be the fibre of the map $Q \rightarrow H$. As $X$ is $(-1)$-connected and $F$ is 2-connected we see that $F \wedge X$ is 2-connected and thus that the map $\pi_{k}(Q \wedge X) \rightarrow \pi_{k}(H \wedge X)=H_{k}(X)$ is an isomorphism for $k \leq 2$.

Next, from the third column in Lemma 4.9 we have an exact sequence

$$
\pi_{2}(Q \wedge X) \rightarrow \pi_{1}\left(\left(S^{1} / 2\right) \wedge X\right) \rightarrow \pi_{1}(X) \rightarrow \pi_{1}(Q \wedge X) \rightarrow \pi_{0}\left(\left(S^{1} / 2\right) \wedge X\right)
$$

The first group here is $H_{2}(X)$, which is zero by assumption. The second is $\pi_{0}((S / 2) \wedge X)$, which is easily shown to be $\pi_{0}(X) / 2$. The fourth is $H_{1}(X)$, and the last is zero for connectivity reasons. We thus have an exact sequence

$$
0 \rightarrow \pi_{0}(X) / 2 \stackrel{\eta}{\rightarrow} \pi_{1}(X) \rightarrow H_{1}(X) \rightarrow 0
$$

as claimed.

Now suppose we have a map $f: X \rightarrow Y$ in $\mathcal{C}$ such that $G(f)$ is an isomorphism. This means that $\pi_{0}(f)=\alpha(G(f))$ and $\pi_{1}(f)=\gamma(G(f))$ are isomorphisms, so $H_{1}(f)$ is also an isomorphism by our short exact sequence. We also know that $H_{0}(f)=\pi_{0}(f)$ by the Hurewicz theorem, and $H_{k}$ vanishes for $k<0$ or $k>1$, so $H_{*}(f)$ is an isomorphism in all degrees. As $X$ and $Y$ are connective this means that $f$ is an equivalence. Thus, the functor $G: \mathcal{C} \rightarrow$ EEED reflects isomorphism.

\section{MOORE SPECTRA}

Lemma 5.1. If $X$ is a Moore spectrum then the Hurewicz map $\pi_{0}(X) \rightarrow H_{0}(X)$ is an isomorphism, and $H_{k}(X)=0$ for all $k \neq 0$.

Proof. We have $\pi_{k}(X)=0$ for $k<0$ by assumption, so the Hurewicz theorem tells us that $H_{k}(X)=0$ for $k<0$ and that $\pi_{0}(X) \simeq H_{0}(X)$. We also have $H_{k}(X)=0$ for $k>0$ by assumption.

Corollary 5.2. The functor $\pi_{0}:$ Moore $\rightarrow$ Ab reflects isomorphism.

Proof. Suppose that $f: X \rightarrow Y$ is a morphism of Moore spectra such that $\pi_{0}(f)$ is an isomorphism, and let $C f$ be the cofibre. Using the lemma we see that $H_{*}(f)$ is an isomorphism, so $H_{*}(C f)=0$. As $X$ and $Y$ are connective, the same is true of $C f$. Lemma 4.11 therefore tells us that $C f$ is contractible, so $f$ is an equivalence. 
Definition 5.3. A free Moore spectrum is a Moore spectrum $X$ for which the group $\pi_{0}(X)$ is free. We write FreeMoore for the category of such spectra.

Lemma 5.4. (a) The functor $\pi_{0}$ : FreeMoore $\rightarrow$ FreeAb is an equivalence.

(b) Every free Moore spectrum is a wedge of some family of copies of $S$.

(c) If $X$ is a free Moore spectrum, then the map $\pi_{0}:[X, Y] \rightarrow \operatorname{Hom}\left(\pi_{0}(X), \pi_{0}(Y)\right)$ is an isomorphism for all $Y$, as is the map $\eta: \pi_{0}(X) / 2 \rightarrow \pi_{1}(X)$.

Proof. Let $X$ be a free Moore spectrum. Choose a family of maps $\left\{g_{i}: S \rightarrow X\right\}_{i \in I}$ giving a basis for the free abelian group $\pi_{0}(X)$. Put $Q=\bigvee_{I} S$ and let $g: Q \rightarrow X$ be given by $g_{i}$ on the $i$ th summand. Let $Y$ be the cofibre of $g$, so $Y$ is connective. By construction $g$ gives an isomorphism from $\pi_{0}(Q)=H_{0}(Q)$ to $\pi_{0}(X)=H_{0}(X)$, and both $Q$ and $X$ have homology concentrated in degree zero, so $H_{*}(Y)=0$. We thus have $Y=0$, so $g$ is an equivalence. This proves (b). Next, the category of those spectra for which (c) holds clearly contains $S$ and is closed under coproducts, so it contains $Q$ and therefore $X$. By specialising to the case where $Y$ is also a free Moore spectrum we obtain (a).

Definition 5.5. A presentation of a Moore spectrum $X$ is a cofibration sequence $P \stackrel{f}{\rightarrow} Q \stackrel{g}{\rightarrow} X \stackrel{h}{\rightarrow} \Sigma P$, where both $P$ and $Q$ are free Moore spectra.

Remark 5.6. As $H_{1}(X)=0$ by the definition of a Moore spectrum, we find that the map $\pi_{0}(f)=H_{0}(f)$ must be injective.

Lemma 5.7. Every Moore spectrum admits a presentation.

Proof. Let $X$ be a Moore spectrum. Choose a free abelian group $Q_{0}$ and a surjective map $g_{0}: Q_{0} \rightarrow \pi_{0}(X)$. By Lemma 5.4 we can find a free Moore spectrum $Q$ with $\pi_{0}(Q)=Q_{0}$, and a map $g: Q \rightarrow X$ with $\pi_{0}(g)=g_{0}$. We can then form a cofibration sequence $P \stackrel{f}{\rightarrow} Q \stackrel{g}{\rightarrow} X \stackrel{h}{\rightarrow} \Sigma P$ for some spectrum $P$. This gives long exact sequences of homotopy and homology groups, showing that $P$ is a Moore spectrum with $\pi_{0}(P)=\operatorname{ker}\left(g_{0}\right)$. This is a subgroup of the free abelian group $Q_{0}$, so it is again free, so $P$ is a free Moore spectrum as required.

Lemma 5.8. If $X$ is a Moore spectrum then the map $\eta: \pi_{0}(X) / 2 \rightarrow \pi_{1}(X)$ is an isomorphism. Thus, the diagram $G(X)$ lies in the subcategory $\mathrm{EMD}^{\prime} \subseteq \mathrm{EEED}$. The corresponding exact Moore diagram $F(X)=$ $E^{-1} G(X)$ is

$$
[S, X] \underset{\rho^{*}}{\stackrel{(\eta \beta)^{*}}{\rightleftarrows}}[S / 2, X]
$$

as in Theorem 1.4.

Proof. Choose a presentation $P \stackrel{f}{\rightarrow} Q \stackrel{g}{\rightarrow} X \stackrel{h}{\rightarrow} \Sigma P$. This gives an exact sequence

$$
\pi_{1}(P) \stackrel{\pi_{1}(f)}{\longrightarrow} \pi_{1}(Q) \rightarrow \pi_{1}(X) \rightarrow \pi_{0}(P) \stackrel{\pi_{0}(f)}{\longrightarrow} \pi_{0}(Q) \rightarrow \pi_{0}(X) \rightarrow 0 .
$$

Here $\pi_{0}(f)$ is injective by the definition of a presentation, so $\pi_{1}(X)$ is the cokernel of $\pi_{1}(f)$, and also $\pi_{0}(X)$ is the cokernel of $\pi_{0}(f)$. Tensoring with $\mathbb{Z} / 2$ is right exact, so $\pi_{0}(X) / 2$ is the cokernel of the map $\pi_{0}(P) / 2 \rightarrow \pi_{0}(Q) / 2$ induced by $\pi_{0}(f)$. We thus have a commutative diagram with right exact rows:

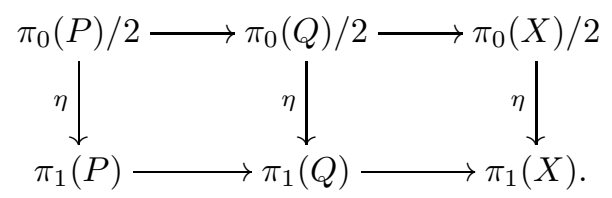

The first two vertical maps are isomorphisms by Lemma 5.4(c), so the third one is also an isomorphism, as claimed. The rest is clear from this.

Lemma 5.9. The functor $F:$ Moore $\rightarrow$ EMD is essentially surjective and reflects isomorphisms. 
Proof. First, let $f: X \rightarrow Y$ be a map of Moore spectra such that $F(f)$ is an isomorphism. Then $\pi_{0}(f)=$ $\alpha(F(f))$ is an isomorphism, so $f$ is an isomorphism by Corollary 5.2. This means that $F$ reflects isomorphism, as claimed. (This could also be deduced from Lemma 4.13.)

Now let $M=(A, B, \phi, \psi)$ be any exact Moore diagram. Choose a surjective homomorphism $g_{0}: Q_{0} \rightarrow A$ with $Q_{0}$ a free abelian group, and let $f_{0}: P_{0} \rightarrow Q_{0}$ be the kernel of $g_{0}$. Note that $P_{0}$ is a subgroup of the free abelian group $Q_{0}$ and so is free. We can thus find a map $f: P \rightarrow Q$ of free Moore spectra with $\pi_{0}(P)=P_{0}$, $\pi_{0}(Q)=Q_{0}$ and $\pi_{0}(f)=f_{0}$. Let $X$ be the cofibre of $f$; we find that $X$ is a Moore spectrum with $\pi_{0}(X)=A$, or in other words $\alpha(F(X))=\alpha(M)$. Corollary 3.20 tells us that the functor $\alpha$ : EMD $\rightarrow$ Ab is full, so we can choose $p: F(X) \rightarrow M$ with $\alpha(p)=1_{A}$. As $\alpha$ reflects isomorphism, we deduce that $p$ is an isomorphism. This proves that $F$ is essentially surjective.

Proposition 5.10. Let $X$ be a Moore spectrum, and let $X^{\prime}$ be an arbitrary spectrum. Then the map

$$
\pi G:\left[X, X^{\prime}\right] \rightarrow \operatorname{ED}\left(\pi G(X), \pi G\left(X^{\prime}\right)\right)
$$

is surjective. The kernel of this map is $K\left(X, X^{\prime}\right)$, and the map $\omega: K\left(X, X^{\prime}\right) \rightarrow \operatorname{Ext}\left(\pi_{0}(X), \pi_{1}\left(X^{\prime}\right)\right)$ is a bijection.

Proof. Note that $G(X) \in \mathrm{EMD}^{\prime}$ by Lemma [5.8, so the map

$$
\alpha: \operatorname{ED}\left(\pi G(X), \pi G\left(X^{\prime}\right)\right) \rightarrow \operatorname{Hom}\left(\pi_{0}(X), \pi_{0}\left(X^{\prime}\right)\right)
$$

is a bijection by Lemma 3.19 It will thus suffice to show that the map $\pi_{0}:\left[X, X^{\prime}\right] \rightarrow \operatorname{Hom}\left(\pi_{0}(X), \pi_{0}\left(X^{\prime}\right)\right)$ is surjective, and to identify its kernel.

We will use the standard notation $N=(A, B, C, \eta, \chi, \psi)$ for $G(X)$ (so $A=\pi_{0}(X)$ and so on), and similarly for $G\left(X^{\prime}\right)$. Choose a presentation $P \stackrel{f}{\rightarrow} Q \stackrel{g}{\rightarrow} X \stackrel{h}{\rightarrow} \Sigma P$, and write $P_{0}=\pi_{0}(P)$ and so on. We then have a diagram

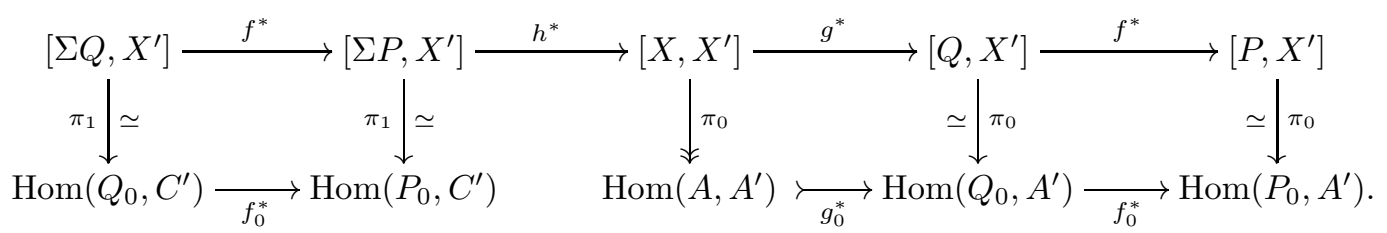

The arrows marked as isomorphisms are indeed isomorphisms, by Lemma 5.4(c). The top row is exact because it comes from a cofibration sequence. The right hand half of the bottom row is left exact, because it arises from the right exact sequence $P_{0} \rightarrow Q_{0} \rightarrow A$. It follows by diagram chasing that the map $\pi_{0}:\left[X, X^{\prime}\right] \rightarrow$ $\operatorname{Hom}\left(A, A^{\prime}\right)$ is surjective, and that the kernel $K\left(X, X^{\prime}\right)$ is the cokernel of the map $f_{0}^{*}: \operatorname{Hom}\left(Q_{0}, C^{\prime}\right) \rightarrow$ $\operatorname{Hom}\left(P_{0}, C^{\prime}\right)$. As the sequence $E=\left(P_{0} \rightarrow Q_{0} \rightarrow A\right)$ is a free resolution of $A$, we see that this cokernel is just $\operatorname{Ext}\left(A, C^{\prime}\right)$. More precisely, we can define $\lambda: \operatorname{Hom}\left(P_{0}, C^{\prime}\right) \rightarrow \operatorname{Ext}\left(A, C^{\prime}\right)$ by $\lambda(u)=u_{*}([E])$, and standard homological algebra says that the sequence

$$
\operatorname{Hom}\left(Q_{0}, C^{\prime}\right) \stackrel{f_{0}^{*}}{\rightarrow} \operatorname{Hom}\left(P_{0}, C^{\prime}\right) \stackrel{\lambda}{\rightarrow} \operatorname{Ext}\left(A, C^{\prime}\right)
$$

is right exact. Consider the diagram

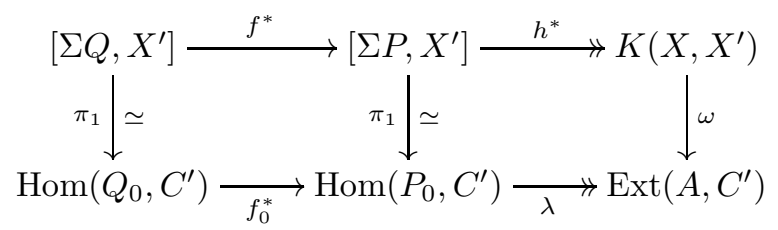

To see that the right hand square commutes, note that $h \in K(X, \Sigma P)$ and $\omega(h)=[E]$. It follows that for $u: \Sigma P \rightarrow X^{\prime}$ we have

$$
\omega\left(h^{*}(u)\right)=\omega(u h)=\omega\left(u_{*} h\right)=\pi_{0}(u)_{*} \omega(h)=\pi_{0}(u)_{*}[E]=\lambda\left(\pi_{0}(u)\right)
$$

as required. As both rows are right exact, we see that $\omega: K(X, Y) \rightarrow \operatorname{Ext}\left(A, C^{\prime}\right)$ is an isomorphism as claimed. 
Corollary 5.11. Let $X$ be a Moore spectrum, and let $X^{\prime}$ be an arbitrary spectrum. Then there is a natural short exact sequence

$$
\text { 2. } \operatorname{Ext}\left(\pi_{0}(X), \pi_{1}\left(X^{\prime}\right)\right) \rightarrow\left[X, X^{\prime}\right] \rightarrow \operatorname{EEED}\left(G(X), G\left(X^{\prime}\right)\right) .
$$

Proof. This follows from the Proposition together with Proposition 4.8 ,

Corollary 5.12. Let $X$ be a $C W$ spectrum with only 0 -cells and 1-cells, and let $X^{\prime}$ be an arbitrary spectrum. Then there is a natural short exact sequence

$$
\text { 2. } \operatorname{Ext}\left(\pi_{0}(X), \pi_{1}\left(X^{\prime}\right)\right) \rightarrow\left[X, X^{\prime}\right] \stackrel{G}{\rightarrow} \operatorname{EEED}\left(G(X), G\left(X^{\prime}\right)\right) .
$$

Proof. The assumption on $X$ is that there exists a cofibration sequence $P \rightarrow Q \rightarrow X \rightarrow \Sigma P$ where $P$ and $Q$ are again free Moore spectra, but the map $\pi_{0}(P) \rightarrow \pi_{0}(Q)$ need not be injective. Let $R_{0}$ and $F_{0}$ be the kernel and image of this map. These are subgroups of the free abelian groups $\pi_{0}(P)$ and $\pi_{0}(Q)$, so they are again free. We have a short exact sequence $R_{0} \rightarrow \pi_{0}(P) \rightarrow F_{0}$, which must split as $F_{0}$ is free. We know that $\pi_{0}$ : FreeMoore $\rightarrow$ FreeAb is an equivalence, so we have a parallel splitting $P=R \vee F$ with $\pi_{0}(R)=R_{0}$ and $\pi_{0}(F)=F_{0}$, and we find that the map $P \rightarrow Q$ is zero on $R$. Now let $Y$ be the cofibre of the map $F \rightarrow Q$. We find that $Y$ is a Moore spectrum, and that $X=Y \vee \Sigma R$. Thus Corollary 5.11 gives the claim for $Y$, and we need only check the claim for $\Sigma R$. As $\pi_{0}(\Sigma R)=0$ the first term in the short exact sequence vanishes, so the claim is just that the map $G:\left[\Sigma R, X^{\prime}\right] \rightarrow \operatorname{EEED}\left(G(\Sigma R), G\left(X^{\prime}\right)\right)$ is bijective. Here $R$ is a free Moore spectrum and so is a wedge of copies of $S$, so we can reduce to the case $R=S$. Here the claim is that $\pi_{1}\left(X^{\prime}\right) \simeq\left[G\left(S^{1}\right), G\left(X^{\prime}\right)\right]$. This is easy to see directly, or one can appeal to the Yoneda Lemma as in Corollary 3.13 ,

We can now prove as promised that $F:$ Moore $\rightarrow$ EMD is an equivalence.

Proof of Theorem 1.4. Suppose that $X$ and $X^{\prime}$ are both Moore spectra. Then 2. $\pi_{1}\left(X^{\prime}\right)=0$ by Lemma 5.8, so 2. $\operatorname{Ext}\left(\pi_{0}(X), \pi_{1}\left(X^{\prime}\right)\right)=0$. Thus, Corollary 5.11tells us that $\left[X, X^{\prime}\right]=\operatorname{EEED}\left(G(X), G\left(X^{\prime}\right)\right)=\operatorname{EMD}\left(F(X), F\left(X^{\prime}\right)\right)$, so $F$ : Moore $\rightarrow$ EMD is full and faithful. It is also essentially surjective by Lemma [5.9] so it is an equivalence.

Corollary 5.13. The functor $G$ : Spectra $\rightarrow$ EEED is essentially surjective.

Here we give a proof using Moore spectra. Proposition 6.2 will give an alternative proof using two-stage Postnikov systems.

Proof. Consider an object $N=(A, B, C, \eta, \chi, \psi) \in$ EEED. Choose Moore spectra $W, X$ and $Y$ with $\pi_{0}(W)=A / 2$ and $\pi_{0}(X)=C$ and $\pi_{0}(Y)=A$. Recall that multiplication by $\eta$ gives an isomorphism $A / 2 \rightarrow \pi_{1}(Y)$. Using Proposition 5.10 and the isomorphism $[\Sigma W, T]=\left[W, \Sigma^{-1} T\right]$ we see that there is a map $f: \Sigma W \rightarrow \Sigma X \vee Y$ such that the induced map $\pi_{1}(f): A / 2 \rightarrow C \oplus A / 2$ is $a \mapsto(\eta(a), a)$. Let $Z$ be the cofibre of $f$. A straightforward calculation with the long exact sequence from the defining cofibration sequence gives $\pi_{0}(Z)=A$ and $\pi_{1}(Z)=C$, with multiplication by $\eta$ being the originally given map $\eta: A \rightarrow C$. This gives an isomorphism $u: \pi N \rightarrow \pi G(Z)$ in ED. We know from Proposition 3.7 that $\pi$ is full, so we can choose $v: N \rightarrow G(Z)$ with $\pi(v)=u$. The same proposition tells us that $\pi$ reflects isomorphism, and $u$ is an isomorphism, so $v$ is an isomorphism. Thus, $N$ is in the essential image of $G$, as required.

We conclude this section by justifying Remark 1.5.

Lemma 5.14. There is an element $\zeta \in \pi_{2}(S / 2)$ with $\beta \zeta=\eta: S^{2} \rightarrow S^{1}$ and $2 \zeta=\rho \eta^{2}$ and $\pi_{2}(S / 2)=(\mathbb{Z} / 4) \zeta$.

This is classical but we include a proof for completeness.

Proof. From the cofibration sequence defining $S / 2$ we obtain an exact sequence

or equivalently

$$
\pi_{2}(S) \stackrel{2}{\rightarrow} \pi_{2}(S) \stackrel{\rho_{*}}{\rightarrow} \pi_{2}(S / 2) \stackrel{\beta_{*}}{\rightarrow} \pi_{1}(S) \stackrel{2}{\rightarrow} \pi_{1}(S)
$$

$$
(\mathbb{Z} / 2) \eta^{2} \stackrel{2=0}{\longrightarrow}(\mathbb{Z} / 2) \eta^{2} \stackrel{\rho_{*}}{\longrightarrow} \pi_{2}(S / 2) \stackrel{\beta_{*}}{\longrightarrow}(\mathbb{Z} / 2) \eta \stackrel{2=0}{\longrightarrow}(\mathbb{Z} / 2) \eta .
$$

It follows that there exists $\zeta$ with $\beta \zeta=\eta$, and that $\pi_{2}(S / 2)=\left\{0, \zeta, \rho \eta^{2}, \zeta+\rho \eta^{2}\right\}$. We also know that $2.1_{S / 2}=\rho \eta \beta$, so $2 \zeta=\rho \eta \beta \zeta=\rho \eta^{2}$, so in fact $\pi_{2}(S / 2)=\{0, \zeta, 2 \zeta, 3 \zeta\}$ and $\zeta$ has order 4 . 
Remark 5.15. There is some indeterminacy in the construction of $\zeta$, but at the end of the argument we see that the only ambiguity is that $\zeta$ could be replaced by $-\zeta$. We do not know of any other approach that eliminates this ambiguity.

Lemma 5.16. For any Moore spectrum $X$ the map $\zeta^{*}:[S / 2, X] \rightarrow \pi_{2}(X)$ is an isomorphism.

Proof. First, if $X=S^{1}$ we have $[S / 2, X]=(\mathbb{Z} / 2) \beta$ and $\pi_{2}(X)=(\mathbb{Z} / 2) \eta=(\mathbb{Z} / 2) \beta \zeta$, so $\zeta^{*}:[S / 2, X] \rightarrow \pi_{2}(X)$ is an isomorphism. Similarly, if $X=S$ then $\pi_{2}(X)=(\mathbb{Z} / 2) \eta^{2}$ and $[S / 2, X]=(\mathbb{Z} / 2) \eta \beta=(\mathbb{Z} / 2)$ and $\pi_{2}(X)=(\mathbb{Z} / 2) \eta^{2}=(\mathbb{Z} / 2) \eta \beta \zeta$, so again $\zeta^{*}:[S / 2, X] \rightarrow \pi_{2}(X)$ is an isomorphism. Now choose a presentation $P \stackrel{f}{\rightarrow} Q \stackrel{g}{\rightarrow} X \stackrel{h}{\rightarrow} \Sigma P$. As $P$ is a wedge of copies of $S$ and $\Sigma P$ is a wedge of copies of $S^{1}$ we find that the maps $\zeta^{*}:[S / 2, P] \rightarrow \pi_{2}(P)$ and $\zeta^{*}:[S / 2, \Sigma P] \rightarrow \pi_{2}(\Sigma P)$ are isomorphisms, and similarly for $Q$. We can now apply the Five Lemma to the diagram

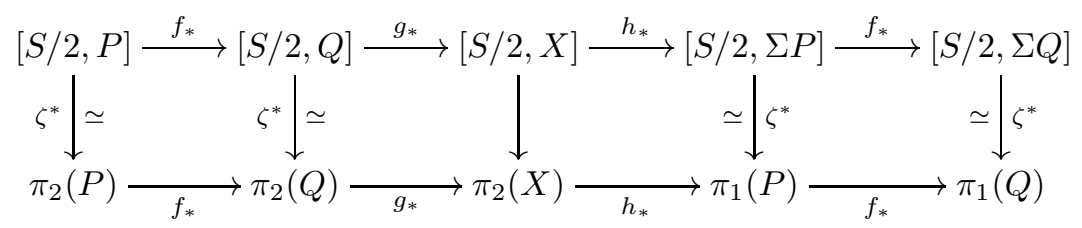

to see that $\zeta^{*}:[S / 2, X] \rightarrow \pi_{2}(X)$ is also an isomorphism.

\section{Two-stage Postnikov systems}

Definition 6.1. We write Postnikov for the category of spectra $X$ such that $\pi_{k}(X)=0$ for $k \notin\{0,1\}$.

Proposition 6.2. The functor $G$ : Postnikov $\rightarrow$ EEED is full and essentially surjective, and it reflects isomorphisms. If $X, X^{\prime} \in$ Postnikov then there is a natural short exact sequence

$$
\text { 2. } \operatorname{Ext}\left(\pi_{0}(X), \pi_{1}\left(X^{\prime}\right)\right) \rightarrow\left[X, X^{\prime}\right] \stackrel{G}{\rightarrow} \operatorname{EEED}\left(G(X), G\left(X^{\prime}\right)\right) .
$$

The proof will follow after some preliminary results.

Lemma 6.3. If $X$ is (-1)-connected then the maps

$$
\left[X, H A^{\prime}\right] \stackrel{G}{\rightarrow} \operatorname{EEED}\left(G(X), G\left(H A^{\prime}\right)\right) \stackrel{\pi}{\rightarrow} \operatorname{ED}\left(\pi G(X), \pi G\left(H A^{\prime}\right)\right) \stackrel{\alpha}{\rightarrow} \operatorname{Hom}\left(\pi_{0}(X), A^{\prime}\right)
$$

are all isomorphisms.

Proof. As $\gamma G\left(H A^{\prime}\right)=\pi_{1}\left(H A^{\prime}\right)=0$, Proposition 3.7 tells us that

$$
\operatorname{EEED}\left(G(X), G\left(H A^{\prime}\right)\right)=\operatorname{ED}\left(\pi G(X), \pi G\left(H A^{\prime}\right)\right),
$$

and using $\gamma G\left(H A^{\prime}\right)=0$ again we see that this is the same as $\operatorname{Hom}\left(\pi_{0}(X), A^{\prime}\right)$. The Universal Coefficient Theorem gives a short exact sequence

$$
\operatorname{Ext}\left(H_{-1}(X), A^{\prime}\right) \rightarrow\left[X, H A^{\prime}\right]=H^{0}\left(X ; A^{\prime}\right) \rightarrow \operatorname{Hom}\left(H_{0}(X), A^{\prime}\right) .
$$

As $X$ is $(-1)$-connected we have $H_{-1}(X)=0$ and $H_{0}(X)=\pi_{0}(X)$ so this collapses to an isomorphism $\left[X, H A^{\prime}\right] \rightarrow \operatorname{Hom}\left(\pi_{0}(X), A^{\prime}\right)=\operatorname{EEED}\left(G(X), G\left(H A^{\prime}\right)\right)$ as required.

Lemma 6.4. If $X$ is (-1)-connected then there is a short exact sequence

$$
\text { 2. } \operatorname{Ext}\left(\pi_{0}(X), C^{\prime}\right) \rightarrow\left[X, \Sigma H C^{\prime}\right] \stackrel{G}{\rightarrow} \operatorname{EEED}\left(G(X), G\left(\Sigma H C^{\prime}\right)\right) .
$$

Proof. In view of Proposition 4.8, it will suffice to give a short exact sequence

$$
\operatorname{Ext}\left(\pi_{0}(X), C^{\prime}\right) \rightarrow\left[X, \Sigma H C^{\prime}\right] \stackrel{\pi G}{\longrightarrow} \mathrm{ED}\left(\pi G(X), \pi G\left(\Sigma H C^{\prime}\right)\right) .
$$

The Universal Coefficient Theorem gives a short exact sequence

$$
\operatorname{Ext}\left(H_{0}(X), C^{\prime}\right) \rightarrow\left[X, \Sigma H C^{\prime}\right] \rightarrow \operatorname{Hom}\left(H_{1}(X), C^{\prime}\right) .
$$


We also know from Lemma 4.13 that $H_{1}(X)$ is the cokernel of the map $\eta: \pi_{0}(X) \rightarrow \pi_{1}(X)$, so $\operatorname{Hom}\left(H_{1}(X), C^{\prime}\right)$ is the same as the group of maps $h$ making the diagram

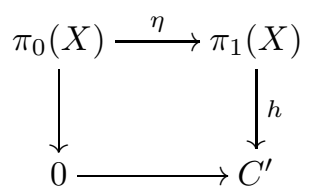

commute, which is $\operatorname{ED}\left(\pi G(X), \pi G\left(\Sigma H C^{\prime}\right)\right)$.

Lemma 6.5. For any abelian groups $A$ and $C$ we have $H_{1} H A=0$ and $H_{2} H A=A / 2$ and $\left[H A, \Sigma^{2} H C\right]=$ $H^{2}(H A ; C)=\operatorname{Hom}(A / 2, C)$. Moreover, if $X$ is the fibre of a map $h: H A \rightarrow \Sigma^{2} H C$ corresponding to a homomorphism $\sigma: A / 2 \rightarrow C$, then the map

$$
\eta: A=\pi_{0}(X) \rightarrow \pi_{1}(X)=C
$$

is just the composite of $\sigma$ with the projection $A \rightarrow A / 2$.

Proof. First, Lemma 4.13 tells us that $H_{1} H A$ is the cokernel of $\eta: \pi_{0} H A \rightarrow \pi_{1} H A$ but $\pi_{1} H A=0$ so $H_{1} H A=0$. For the second claim, we reuse the spectrum $Q$ from Lemma 4.9. As the fibre of the map $Q \rightarrow H$ is 2-connected we see that $H_{2} H A=\pi_{2}(Q \wedge H A)=H_{2}(Q ; A)$. The cofibration sequence $S \rightarrow Q \rightarrow S^{2} / 2 \rightarrow S^{1}$ gives $H_{2}(Q ; A)=H_{2}\left(S^{2} / 2 ; A\right)=H_{0}(S / 2 ; A)$, and the Universal Coefficient Theorem gives a short exact sequence

$$
H_{0}(S / 2) \otimes A \rightarrow H_{0}(S / 2 ; A) \rightarrow \operatorname{Tor}\left(H_{-1}(S / 2), A\right),
$$

which collapses to an isomorphism $H_{0}(S / 2 ; A)=A / 2$. Finally, the cohomological UCT gives a short exact sequence

$$
\operatorname{Ext}\left(H_{1} H A, C\right) \rightarrow H^{2}(H A ; C) \rightarrow \operatorname{Hom}\left(H_{2} H A, C\right)
$$

which collapses to an isomorphism $H^{2}(H A ; C)=\operatorname{Hom}\left(H_{2} H A, C\right)=\operatorname{Hom}(A / 2, C)$ as claimed.

We now see in particular that $\left[H, \Sigma^{2} H / 2\right]=\mathbb{Z} / 2$, so there is a unique nontrivial map $h_{1}: H \rightarrow \Sigma^{2} H / 2$. We can also compose the projection $H \rightarrow H / 2$ with the Steenrod operation $\mathrm{Sq}^{2}: H / 2 \rightarrow \Sigma^{2} H / 2$ to get a map $h_{2}: H \rightarrow \Sigma^{2} H / 2$. Standard calculations show that the reduced cohomology of $\mathbb{C} P^{2}$ is $\mathbb{Z}\left\{x, x^{2}\right\}$ with $|x|=2$, and $\mathrm{Sq}^{2}$ sends the mod 2 reduction of $x$ to the $\bmod 2$ reduction of $x^{2}$. This shows that $h_{2} \neq 0$ and so $h_{2}=h_{1}$. Moreover, $\mathbb{C} P^{2}$ is just the cofibre of $\eta: S^{3} \rightarrow S^{2}$, so $S / \eta=\Sigma^{-2} \mathbb{C} P^{2}$. The above calculation therefore gives a commutative diagram as follows:

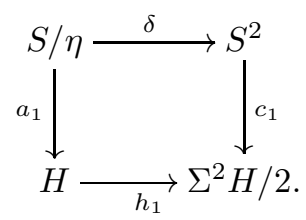

Now suppose we have a general map $h: H A \rightarrow \Sigma^{2} H C$, corresponding to $\sigma: A / 2 \rightarrow C$, and we form a cofibration sequence

$$
\Sigma H C \stackrel{f}{\rightarrow} X \stackrel{g}{\rightarrow} H A \stackrel{h}{\rightarrow} \Sigma^{2} H C .
$$

Consider an element $a \in A$ and the corresponding element $c=\sigma(a+2 A) \in C$ (which automatically has $2 c=0)$. We have a map $\mu_{a}: \mathbb{Z} \rightarrow A$ given by $\mu_{a}(n)=n a$, and a map $\nu_{c}: \mathbb{Z} / 2 \rightarrow C$ given by $\nu_{c}(1)=c$. Consider the following diagram:

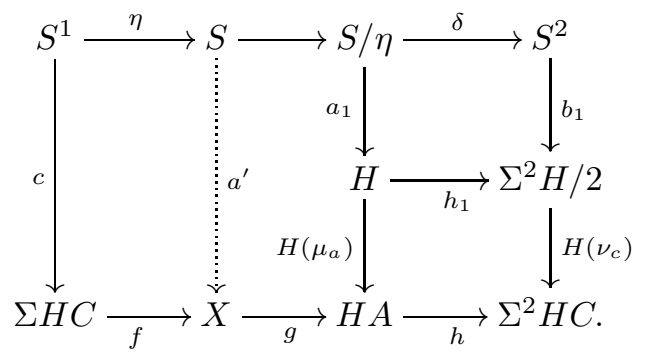


We have just shown that the top right square commutes. As our identification of $\left[H A, \Sigma^{2} H C\right]$ is natural, the bottom right square commutes as well. As the top and bottom rows are cofibration sequences, we can choose $a^{\prime}$ making everything commute. If we use $f_{*}$ to identify $\pi_{0}(X)$ with $A$, and $g_{*}$ to identify $\pi_{1}(X)$ with $C$, then the conclusion is that $\eta a=c$. This shows that $\eta: \pi_{0}(X) \rightarrow \pi_{1}(X)$ is essentially the same as $\sigma$, as claimed.

Lemma 6.6. Suppose that $X$ is (-1)-connected and $X^{\prime} \in$ Postnikov. Then there is a left exact sequence

$$
\operatorname{Ext}\left(\pi_{0}(X), \pi_{1}\left(X^{\prime}\right)\right) \rightarrow\left[X, X^{\prime}\right] \stackrel{\pi G}{\longrightarrow} \operatorname{ED}\left(G(X), G\left(X^{\prime}\right)\right) .
$$

Proof. We will use the notation

$$
\begin{aligned}
M & =\pi G(X)=(A \stackrel{\eta}{\rightarrow} C) \\
M^{\prime} & =\pi G\left(X^{\prime}\right)=\left(A^{\prime} \stackrel{\eta}{\rightarrow} C^{\prime}\right) .
\end{aligned}
$$

Put $A^{\prime}=\pi_{0}\left(X^{\prime}\right)$ and $C^{\prime}=\pi_{1}\left(X^{\prime}\right)$, so we have a Postnikov cofibration sequence

$$
\Sigma H C^{\prime} \stackrel{f}{\rightarrow} X^{\prime} \stackrel{g}{\rightarrow} H A^{\prime} \stackrel{h}{\rightarrow} \Sigma^{2} H C^{\prime} .
$$

(This is usually constructed as a fibration sequence, but in the stable category a fibration sequence can be converted to a cofibration sequence by changing the sign of any one of the maps.) This gives a commutative diagram

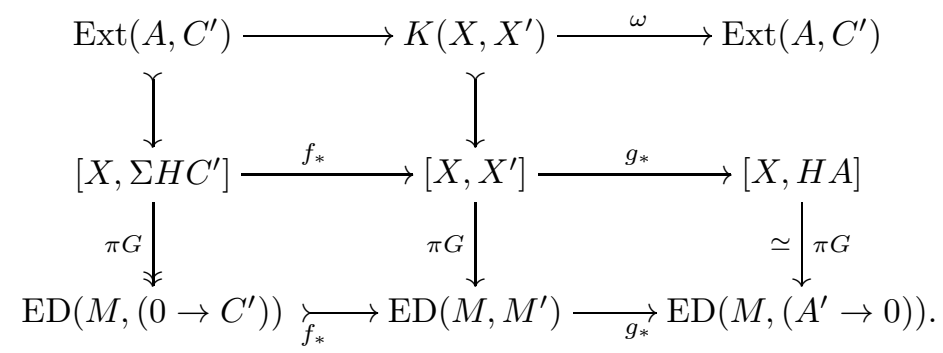

The middle row is exact as it arises from a cofibration sequence. The bottom row is left exact by inspection of the definitions. The first column is short exact by Lemma 6.4. The middle column is left exact by definition of $K\left(X, X^{\prime}\right)$. The map $[X, H A] \rightarrow \mathrm{ED}\left(M, A^{\prime} \rightarrow 0\right)$ is an isomorphism by Lemma 6.3. We leave it to the reader to check that the composite of the top row is the identity. It follows that the map $\operatorname{Ext}\left(A, C^{\prime}\right) \rightarrow K\left(X, X^{\prime}\right)$ is injective, and by chasing the diagram we see that it is also surjective.

Proof of Proposition 6.2. Suppose that $X, X^{\prime} \in$ Postnikov, and use the usual notation $A=\pi_{0}(X)$ and so on. Suppose we are given a commutative square

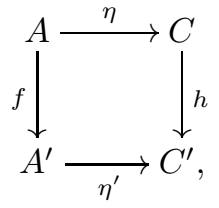

corresponding to a morphism $(f, h) \in \operatorname{ED}\left(\pi G(X), \pi G\left(X^{\prime}\right)\right)$. Consider the following diagram:

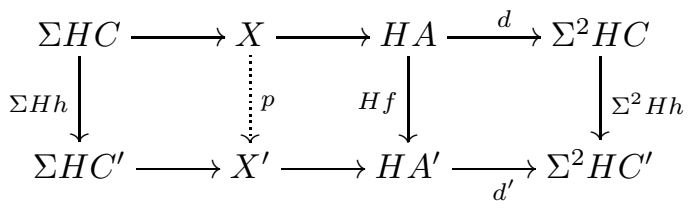

The rows are the standard Postnikov cofibration sequences for $X$ and $X^{\prime}$. As $d$ and $d^{\prime}$ correspond to $\eta$ and $\eta^{\prime}$ as in Lemma 6.5. we see that the right hand square commutes, so we can choose $p$ making everything commute; we then have $\pi G(p)=(f, h)$. This shows that $\pi G$ : Postnikov $\rightarrow$ ED is full. If $\pi G(p)$ is an isomorphism then $\pi_{k}(p)$ is an isomorphism for $k \in\{0,1\}$ (by assumption) and also for $k \notin\{0,1\}$ (as in that case $\pi_{k}(X)=0=\pi_{k}\left(X^{\prime}\right)$ ); so $p$ is an equivalence. This shows that $\pi G$ reflects isomorphism, and a fortiori 
$G$ has the same property. Next, given an object $(A \stackrel{\eta}{\rightarrow} C) \in$ ED we note that $\eta$ factors through $A / 2$ and so gives a map $h: H A \rightarrow \Sigma^{2} H C$. The fibre $F h$ is then an object of Postnikov with $\pi G(F h) \simeq(A \rightarrow C)$; so $\pi G$ : Postnikov $\rightarrow$ EEED is essentially surjective.

We also see from Lemma [6.6 that the map $\omega: K\left(X, X^{\prime}\right) \rightarrow \operatorname{Ext}\left(A, C^{\prime}\right)$ is an isomorphism. We deduce using Proposition 4.8 that there is a natural short exact sequence

$$
\text { 2. } \operatorname{Ext}\left(A, C^{\prime}\right) \rightarrow\left[X, X^{\prime}\right] \rightarrow \operatorname{EEED}\left(G(X), G\left(X^{\prime}\right)\right) .
$$

In particular, the functor $G$ : Postnikov $\rightarrow$ EEED is full.

Finally, suppose we have an object $N \in$ EEED. We have seen that $\pi G$ is essentially surjective, so we can choose $X \in$ Postnikov and an isomorphism $p_{0}: \pi G(X) \rightarrow \pi N$. As $\pi$ is full we can choose $p: G(X) \rightarrow N$ lifting $p_{0}$, and as $\pi$ reflects isomorphisms we see that $p$ is an isomorphism. This proves that $G$ is essentially surjective.

\section{Duality}

We next discuss two kinds of duality for the category EEED.

Lemma 7.1. Let $\mathcal{J}$ be as in Definition 3.11. Then there is a functor $\Delta: \mathcal{J}^{o p} \rightarrow \mathcal{J}$ given by

$$
\begin{array}{lll}
\Delta(a)=c & \Delta(b)=b & \Delta(c)=S \\
\Delta(\rho)=\beta & \Delta(\eta)=\eta & \Delta(\beta)=\rho .
\end{array}
$$

Moreover, this satisfies $\Delta^{2}=1$.

Proof. A straightforward check of definitions.

This is connected with topology as follows:

Lemma 7.2. If we define $\Xi:(\mathcal{J} \times \mathcal{J})^{o p} \rightarrow \mathrm{Ab}$ by $\Xi(x, y)=\left[T(x) \wedge T(y), S^{1}\right]$, then there are natural isomorphisms

$$
\mathcal{J}(x, \Delta(y)) \simeq \Xi(x, y) \simeq \mathcal{J}(y, \Delta(x)) .
$$

Proof. It is equivalent to claim that there are natural isomorphisms $T(\Delta(x)) \simeq F\left(T(x), S^{1}\right)$. This is clear for $x \in\{a, c\}$ (so $T(x) \in\left\{S, S^{1}\right\}$ ). Next, we can apply $F\left(-, S^{1}\right)$ to the cofibration sequence

$$
S \stackrel{2}{\rightarrow} S \stackrel{\rho}{\rightarrow} S / 2 \stackrel{\beta}{\rightarrow} S^{1} \stackrel{2}{\rightarrow} S^{1}
$$

to get a fibration sequence

$$
S \stackrel{2}{\rightarrow} S \stackrel{\beta^{*}}{\rightarrow} F\left(S / 2, S^{1}\right) \stackrel{\rho^{*}}{\rightarrow} S^{1} \stackrel{2}{\rightarrow} S^{1} .
$$

In principle we need to change a sign to convert this fibration to a cofibration, but $\rho=-\rho$ and $\beta=-\beta$ so we need not worry about this. It follows from the essential uniqueness of cofibration sequences that there is an equivalence $\nu: S / 2 \rightarrow F\left(S / 2, S^{1}\right)$ making the diagram on the left commute:
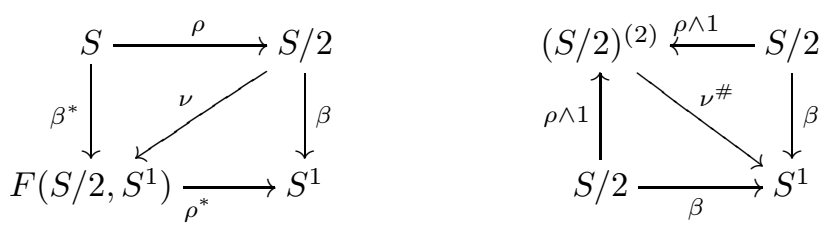

(One can check that $\nu$ has order 4 , and that the only indeterminacy is that we could replace $\nu$ by $-\nu$.) The map $\nu$ is adjoint to a map $\nu^{\#}: S / 2 \wedge S / 2 \rightarrow S^{1}$, and the adjoint form of the left hand diagram is shown on the right. It follows that everything fits together as described.

We can use this to define a Brown-Comenetz type duality as follows.

Definition 7.3. For $N \in \mathrm{EED}=\left[\mathcal{J}^{\mathrm{op}}, \mathrm{Ab}\right]$ we define $J N: \mathcal{J}^{\mathrm{op}} \rightarrow$ Ab by $J N(x)=\operatorname{Hom}(N(\Delta x), \mathbb{Q} / \mathbb{Z})$. More explicitly, we have

$$
J(B \stackrel{\psi}{\rightarrow} A \stackrel{\eta}{\rightarrow} C \stackrel{\chi}{\rightarrow} B)=\left(B^{*} \stackrel{\chi^{*}}{\rightarrow} C^{*} \stackrel{\eta^{*}}{\rightarrow} A^{*} \stackrel{\psi^{*}}{\rightarrow} B^{*}\right)
$$

(where $U^{*}$ denotes $\operatorname{Hom}(U, \mathbb{Q} / \mathbb{Z})$ ). 
Remark 7.4. As the group $\mathbb{Q} / \mathbb{Z}$ is divisible, the functor $U \mapsto U^{*}$ is exact, so the functor $J:$ EED $^{\text {op }} \rightarrow$ EED preserves EEED. It is also clear that there is a natural map $N \rightarrow J^{2}(N)$ which is an isomorphism when the groups $A, B$ and $C$ are all finite.

Proposition 7.5. Let $I X$ denote the Brown-Comenetz dual of a spectrum $X$, which is characterised by a natural isomorphism $[W, I X] \simeq \operatorname{Hom}\left(\pi_{0}(W \wedge X), \mathbb{Q} / \mathbb{Z}\right)$ for all spectra $W$. Then $G(\Sigma I X)=J(G(X))$.

Proof. For $u \in \mathcal{J}$ we have

$$
G(\Sigma I X)(u)=\left[\Sigma^{-1} T(u), I X\right]=\operatorname{Hom}\left(\pi_{0}\left(\Sigma^{-1} T(u) \wedge X\right), \mathbb{Q} / \mathbb{Z}\right) .
$$

We also have $T(u)=T\left(\Delta^{2} u\right)=F\left(T(\Delta u), S^{1}\right)$, so $\Sigma^{-1} T(u) \wedge X=F(T(\Delta u), X)$, so

$$
\pi_{0}\left(\Sigma^{-1} T(u) \wedge X\right)=[T(\Delta u), X]=G(X)(\Delta u) .
$$

Putting this together gives $G(\Sigma I X)(u)=\operatorname{Hom}(G(X)(\Delta u), \mathbb{Q} / \mathbb{Z})=J(G(X))(u)$.

We now discuss a different construction that is more analogous to Spanier-Whitehead duality. It does not work very well, but it is interesting that it works at all.

Definition 7.6. Recall that $F_{x}$ denotes the representable functor $\mathcal{J}(-, x): \mathcal{J}^{\mathrm{op}} \rightarrow$ Ab. We define $\Delta: \mathrm{EED}^{\mathrm{op}} \rightarrow$ EED by

$$
\Delta(N)(x)=\operatorname{EED}\left(N, F_{\Delta(x)}\right) .
$$

Note that a map $M \rightarrow \Delta N$ consists of a natural system of maps $M(x) \rightarrow \operatorname{EED}\left(N, F_{\Delta(x)}\right)$, or equivalently a natural system of maps $M(x) \rightarrow \operatorname{Hom}(N(y), \mathcal{J}(y, \Delta(x)))$, or equivalently a natural system of maps

$$
M(x) \otimes N(y) \rightarrow \Xi(x, y) .
$$

Using this we see that $\operatorname{EED}(M, \Delta(N)) \simeq \operatorname{EED}(N, \Delta(M))$, so $\Delta$ is its own adjoint. In particular, we have natural maps

$$
M(x) \otimes(\Delta M)(y)=M(x) \otimes \operatorname{EEED}\left(M, F_{\Delta y}\right) \stackrel{1 \otimes \mathrm{eval}_{x}}{\longrightarrow} M(x) \otimes \operatorname{Hom}(M(x), \Xi(y, x)) \stackrel{\text { eval }}{\longrightarrow} \Xi(y, x) \simeq \Xi(x, y)
$$

and these correspond to a natural map $\kappa: M \rightarrow \Delta^{2} M$. If we regard this as a morphism in EED, this is the unit of our adjunction; if we regard it instead as a morphism $\Delta^{2} M \rightarrow M$ in $\mathrm{EED}^{\mathrm{op}}$, it is the counit.

We can also use the Yoneda lemma to see that

$$
\Delta\left(F_{x}\right)(y)=\operatorname{EED}\left(F_{x}, F_{\Delta(y)}\right)=F_{\Delta(y)}(x)=\mathcal{J}(x, \Delta(y)) \simeq \mathcal{J}(y, \Delta(x))=F_{\Delta(x)}(y)
$$

so $\Delta\left(F_{x}\right)=F_{\Delta(x)}$.

Proposition 7.7. There is a natural map $G\left(F\left(X, S^{1}\right)\right) \rightarrow \Delta(G(X))$, which is an isomorphism when $X$ is any wedge of copies of $S, S^{1}$ and $S / 2$.

Proof. Using the evaluation map ev: $F\left(X, S^{1}\right) \wedge X \rightarrow S^{1}$ we get maps

$$
\begin{aligned}
G\left(F\left(X, S^{1}\right)\right)(x) \otimes G(X)(y) & =\left[T(x), F\left(X, S^{1}\right)\right] \otimes[T(y), X] \\
& \rightarrow\left[T(x) \wedge T(y), F\left(X, S^{1}\right) \wedge X\right] \\
& \stackrel{\mathrm{ev}_{*}}{\longrightarrow}\left[T(x) \wedge T(y), S^{1}\right]=\Xi(x, y) .
\end{aligned}
$$

As explained above this gives a map $\kappa_{X}: G\left(F\left(X, S^{1}\right)\right) \rightarrow \Delta(G(X))$. In particular, when $X=T(u) \in$ $\left\{S, S^{1}, S / 2\right\}$ we have $F\left(X, S^{1}\right)=T(\Delta(u))$ so $\kappa_{X}$ is a map from $G(T(\Delta u))=F_{\Delta u}$ to $\Delta\left(F_{u}\right)$; we leave it to the reader to check that this is the same as the isomorphism established previously. Moreover, the domain and codomain of $\kappa$ are both functors that convert wedges to products, so we see that $\kappa_{X}$ is an isomorphism when $X$ is any wedge of copies of $S, S^{1}$ and $S / 2$.

We can make the functor $\Delta$ more explicit as follows: 
Proposition 7.8. If $N=(A, B, C, \eta, \chi, \psi) \in$ EED then $\Delta(N) \simeq\left(A^{\prime}, B^{\prime}, C^{\prime}, \eta^{\prime}, \chi^{\prime}, \psi^{\prime}\right)$, where $B^{\prime}=$ $\operatorname{Hom}(B, \mathbb{Z} / 4)$, and $A^{\prime}$ is the set of pairs $(g, h)$ making the left hand diagram below commute, and $C^{\prime}$ is the set of pairs $(f, g)$ making the right hand diagram commute.
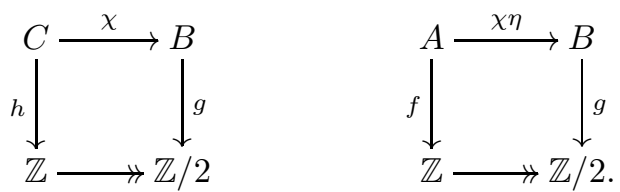

There is a unique nonzero homomorphism $t: \mathbb{Z} / 2 \rightarrow \mathbb{Z} / 4$ and a unique nonzero homomorphism $s: \mathbb{Z} / 4 \rightarrow$ $\mathbb{Z} / 2$, and using these we can describe $\eta^{\prime}, \chi^{\prime}$ and $\phi^{\prime}$ as follows:

$$
\begin{aligned}
\psi^{\prime}(g) & =(s g, 0) \\
\eta^{\prime}(g, h) & =(0, g) \\
\chi^{\prime}(f, g) & =t g .
\end{aligned}
$$

Proof.

By definition we have

$$
A^{\prime}=\Delta(N)(a)=\operatorname{EED}\left(N, F_{\Delta(a)}\right)=\operatorname{EED}\left(N, F_{c}\right) .
$$

Looking back to Corollary 3.13 , we see that this is the set of triples $(f, g, h)$ making the following diagram commute:

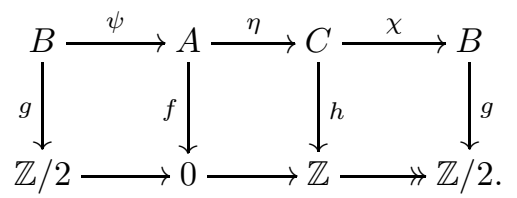

Of course $f$ must be zero and the first square commutes automatically. Note also that for any homomorphism $h: C \rightarrow \mathbb{Z}$ we have $2 h \eta=h \circ(2 \eta)=0$ but $\mathbb{Z}$ is torsion free so $h \eta=0$. Thus, the second square also commutes automatically and we need only consider the third one. This gives the stated description of $A^{\prime}$.

Similarly, $C^{\prime}$ is the set of triples $(f, g, h)$ making the diagram

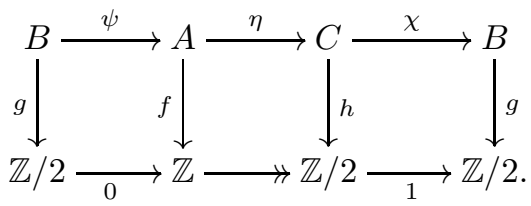

The first square commutes automatically because $2 \psi=0$. The third square forces $h$ to be $g \chi$, so we need not mention $h$ explicitly. The only remaining condition is that $g \chi \eta$ should be the mod two reduction of $f$, which is the stated description of $C^{\prime}$.

Next, $B^{\prime}$ is the set of triples $(f, g, h)$ making the diagram on the left (and therefore also the diagram on the right) commute:
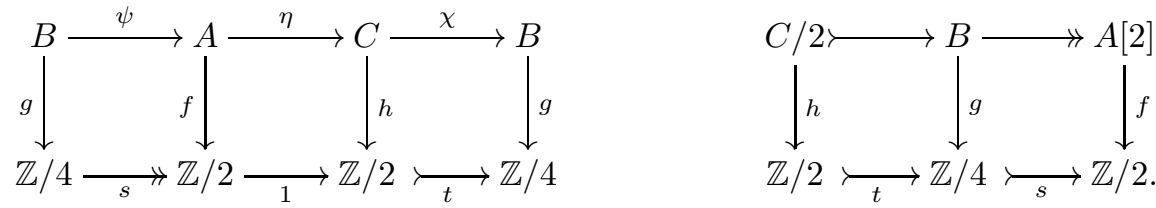

There is a projection $\pi: B^{\prime} \rightarrow \operatorname{Hom}(B, \mathbb{Z} / 4)$ given by $(f, g, h) \mapsto g$, and we claim that this is bijective. First, suppose that $g=0$. The left half of the right hand diagram shows that $h=0$, and then the middle square of the left diagram shows that $f=h \eta=0$. This proves that $\pi$ is injective. Suppose instead that we start with an arbitrary map $g: B \rightarrow \mathbb{Z} / 4$. As $2 \chi=0$ we have $2 g \chi=0$ so the map $g \chi: C \rightarrow \mathbb{Z} / 4$ factors through $(\mathbb{Z} / 4)[2]=t(\mathbb{Z} / 2)$. Thus, there is a unique map $h: C \rightarrow \mathbb{Z} / 2$ with $g \chi=t h$, so the right square of the left diagram commutes. We define $f=h \eta: A \rightarrow \mathbb{Z} / 2$, which makes the middle square commute. We now have $t f \psi=t h \eta \psi=g \chi \eta \psi$, and $\chi \eta \psi=2.1_{B}$ so $t f \psi=2 g$. On the other hand, $t s=2: \mathbb{Z} / 4 \rightarrow \mathbb{Z} / 4$, so we also have 
$t s g=2 g$. As $t$ is injective and $t f \psi=2 g=t s g$ we conclude that $f \psi=s g$, so the left square commutes. We thus have an element $(f, g, h) \in B^{\prime}$ with $\pi(f, g, h)=g$, showing that $\pi$ is actually an isomorphism. We will therefore identify $B^{\prime}$ with $\operatorname{Hom}(B, \mathbb{Z} / 4)$.

Now consider the picture

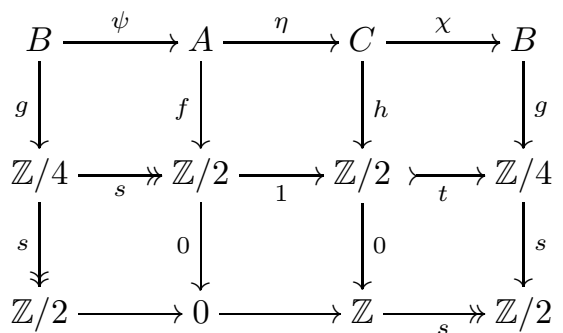

The second and third rows are $F_{\Delta(b)}=F_{b}$ and $F_{\Delta(a)}=F_{c}$, and the map between them is $F_{\Delta(\rho)}=F_{\beta}$. The map from the first to the second row defines a general element of $B^{\prime}=\operatorname{EED}\left(N, F_{b}\right)$. The map $\psi^{\prime}: B^{\prime} \rightarrow A^{\prime}$ is defined by composing vertically to give the system of maps $0: A \rightarrow 0, s g: B \rightarrow \mathbb{Z} / 2$ and $0: C \rightarrow \mathbb{Z}$. In terms of our slightly more compact notation for elements of $B^{\prime}$ and $A^{\prime}$, the formula is $\psi^{\prime}(g)=(s g, 0)$ as claimed. We leave it to the reader to check the formulae $\eta^{\prime}(g, h)=(0, g)$ and $\chi^{\prime}(f, g)=t g$ in the same way.

Remark 7.9. It follows easily from the above that the torsion subgroups in $A^{\prime}$ and $C^{\prime}$ have exponent 2 (or are trivial). Thus, if $A$ or $C$ has any elements of order greater than 2, we see that $N \nsucceq \Delta^{2}(N)$. In fact, if $A$ and $C$ are finite groups of odd order (which forces the structure maps to be zero, and $B$ to be the zero group) then $\Delta(N)=0$.

Remark 7.10. Consider an object $N=(A, B, C, \eta, \chi, \psi) \in$ EEED. Suppose that $A$ and $C$ are elementary abelian groups of finite rank, and that $\eta: A \rightarrow C$ is zero. We then see from Proposition 3.17 that $B=A \oplus C$, and Proposition 7.8 gives $A^{\prime}=\operatorname{Hom}(A, \mathbb{Z} / 2)$ and $B^{\prime}=C^{\prime}=\operatorname{Hom}(A, \mathbb{Z} / 2) \oplus \operatorname{Hom}(C, \mathbb{Z} / 2)$. Just by comparing orders we deduce that the sequence $C=C / 2 \rightarrow B \rightarrow A[2]=A$ cannot be short exact, so $\Delta(N) \notin$ EEED. Thus, the functor $\Delta:$ EED $^{\mathrm{op}} \rightarrow$ EED does not preserve the category EEED.

\section{REFERENCES}

[1] Hans J. Baues, Algebraic homotopy, Cambridge Studies in Advanced Mathematics, vol. 15, Cambridge University Press, 1989.

[2] Hans-Joachim Baues, Homotopy type and homology, Oxford Mathematical Monographs, The Clarendon Press Oxford University Press, New York, 1996. Oxford Science Publications. MR1404516 (97f:55001)

[3] Peter Freyd, Stable homotopy, Proc. conf. categorical algebra (La Jolla, Calif., 1965), 1966, pp. 121-172. MR35\#2280

[4] Nicolae Popesco and Pierre Gabriel, Caractérisation des catégories abéliennes avec générateurs et limites inductives exactes, C. R. Acad. Sci. Paris 258 (1964), 4188-4190 (French). MR0166241 (29 \#3518)

[5] Hirosi Toda, Order of the identity class of a suspension space, Ann. of Math. (2) 78 (1963), 300-325. MR0156347 (27 \#6271)

School of Mathematics and Statistics, University of Sheffield, The Hicks Building, Hounsfield Road, Sheffield, S3 7RH, UK

E-mail address: N.P.Strickland@sheffield.ac.uk

$U R L:$ http://www.shef.ac.uk/nps 